\title{
The Parametric Aircraft Noise Analysis Module - status overview and recent applications
}

\author{
Lothar Bertsch* Sébastien Guérin† Gertjan Looye $\ddagger$ and Michael Pott-Pollenske* \\ German Aerospace Center (DLR)
}

\begin{abstract}
The German Aerospace Center (DLR) is investigating aircraft noise prediction and noise reduction capabilities. The Parametric Aircraft Noise Analysis Module (PANAM) is a fast prediction tool by the DLR Institute of Aerodynamics and Flow Technology to address overall aircraft noise. It was initially developed to (1) enable comparative design studies with respect to overall aircraft ground noise and to (2) indentify promising low-noise technologies at early aircraft design stages. A brief survey of available and established fast noise prediction codes is provided in order to rank and classify PANAM among existing tools. PANAM predicts aircraft noise generated during arbitrary $3 \mathrm{D}$ approach and take-off flight procedures. Noise generation of an operating aircraft is determined by its design, the relative observer position, configuration settings, and operating condition along the flight path. Feasible noise analysis requires a detailed simulation of all these dominating effects. Major aircraft noise components are simulated with individual models and interactions are neglected. Each component is simulated with a separate semi-empirical and parametric noise source model. These models capture major physical effects and correlations yet allow for fast and accurate noise prediction. Sound propagation and convection effects are applied to the emitting noise source in order to transfer static emission into aircraft ground noise impact with respect to the actual flight operating conditions. Recent developments and process interfaces are presented and prediction results are compared with experimental data recorded during DLR flyover noise campaigns with an Airbus A319 (2006), a VFW614 (2009), and a Boeing B737-700 (2010). Overall, dominating airframe and engine noise sources are adequately modeled and overall aircraft ground noise levels can sufficiently be predicted. The paper concludes with a brief overview on current code applications towards selected noise reduction technologies.
\end{abstract}

\section{Nomenclature}

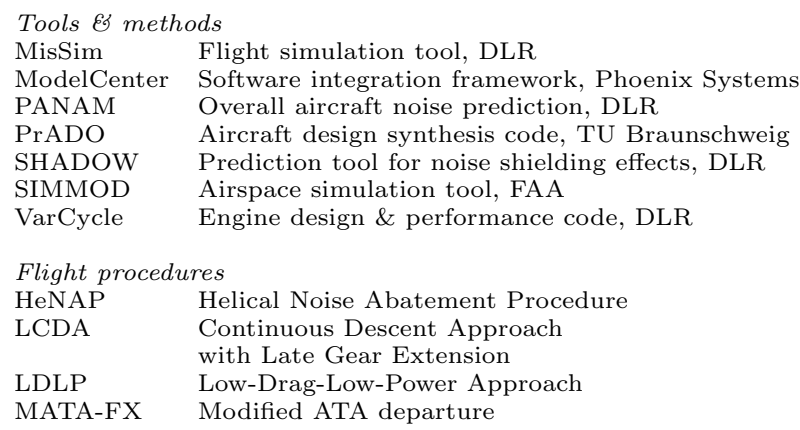




\section{Introduction}

DLR is investigating overall aircraft noise prediction for application within the design process of new vehicle concepts. Noise prediction capability is of great importance when it comes to the design of new aircraft configurations or the evaluation of low-noise design modifications to existing concepts. Early within the design process of new aircraft concepts, i.e. conceptual phase, major aircraft and engine design parameters are still subject to change. At this point key parameters, e.g. wing span, are determined which drive the final aircraft concept. Comprehensive surveys dedicated to airframe noise research activities and methodologies emphasize the requirement to predict overall aircraft noise in the vehicle design stage, i.e. Farassat ${ }^{1}$ in 2006 and Dobrzynski ${ }^{2}$ in 2010. The parameter setting is optimized for best performance according to the overall aircraft requirements. Identification of an optimal setting completes the conceptual aircraft design phase. The selected key parameters are kept constant during subsequent detailed design phases. Therefore, it is inevitable and imperative ${ }^{1}$ to implement noise as a new constraint already within the conceptual design phase. The quantity and complexity of required input data for the noise prediction are limited within conceptual design hence impose further restrictions on a noise prediction tool. Moreover, such noise prediction method requires a modular setup to allow for direct implementation into existing multidisciplinary, conceptual aircraft design codes. Obviously, low computational costs are inevitable when noise prediction is embedded within multidisciplinary processes. For such an application, the use of high fidelity methods is ruled out and fast prediction methods are required ${ }^{2}$.

Existing fast overall aircraft noise prediction tools can be separated into two groups, referred to as restricted and parametric prediction methodologies. The first group works with fully empirical approximations derived from measurements. Therefore, application is limited to existing technology and configuration and design settings as coped by the underlying experimental data. These methods evaluate measured ground noise levels and subtract modeled propagation effects in order to simulate the originating sound source. Usually, such tools have a commercial or corporate background with the focus on existing technology to maximize the potential user group (e.g. airports, airlines, legislation) and increase sales/contracts in order to compensate the development costs. Tools from the second group pursue a some-what empirical approach but are more physics-based in order to expand prediction capabilities beyond existing aircraft configurations and allow for parameter variations. These prediction methods start with the theory of modelling the noise source emission to ultimately propagate the noise onto the ground. Usually, the noise generation is separated into engine and airframe component contributions. Due to the required dedicated noise measurements and the increased scientific complexity, these tools are more likely to be found in state-subsidized environments such as research institutions and universities. Most aircraft manufacturers operate their own confidential noise prediction tools. Due to the lack of information these tools can not be allocated into one specific group. Probably these tools are highly empirical based on a huge available data base and furthermore customized for each specific aircraft and engine type under consideration. It can be expected that these tools are parametric to some extend in order to account for configurational and operational noise generating effects as well.

\section{A. Existing tools}

Restricted prediction methodologies are fully empirical methods based on databases with measured and standardized noise levels for many aircraft and engine types. As a consequence, only existing aircraft and engine technology can be evaluated with these tools ${ }^{\mathrm{a}}$. The tools are developed for best agreement of predicted absolute levels compared with flyover measurement recordings and at the same time to guarantee low computational requirements. Some tools use simple methods to account for directivity effects, others incoperate noise directivity within their underlying data bases. Effects of observer distance and vehicle velocity are accounted for by simple modifications to the stored data. The applied approximations and methods are standardized, e.g. Noise-Power-Distance-Tables, and can be found in corresponding literature ${ }^{3-5}$. Usually, the overall noise cannot be separated into individual contributions hence componential noise evaluation is not possible. Tools out of this group are designed to evaluate medium to long term average noise levels around airports rather than single flyover events ${ }^{6}$. Best agreement to experimental data can be achieved if multiple flyover events and airspace scenarios are evaluated because most details are smeared out due to time integration and extensive averaging.

In general, the results can be accurate to approximately \pm 1 to $\pm 2 \mathrm{~dB}^{5,7}$ for observers located directly

${ }^{a}$ maybe new techology could be accounted for by modification of the underlying databases provided as input for these tools 
along the flight ground track with the aircraft operating in lower altitudes hence these tools can qualify for certification and legislation processes. Although, if predicting long-term average noise contours even these small variations can result in up to $40 \%$ (for $\pm 1 \mathrm{~dB}$ ) or $80 \%$ (for $\pm 2 \mathrm{~dB}$ ) area error according to Smith $^{8}$. Result accuracy is significantly reduced for observers located off-side the ground flight track, for low angles of incidence, and with increasing distance between aircraft and observer ${ }^{7}$. Due to the amount of data, only representative operating conditions, are stored and can be accounted for. Therefore, each flight path has to be separated into a combination of existing constant flight segments, hence the considered flight operations should be rather similar to existing procedures stored in the database. Obviously, the complex schedule of configurational changes along realistic approach procedures cannot be accounted for at all. Departure procedures have to be modeled with constant thrust ${ }^{\mathrm{b}}$ setting although most airlines use varying or minimum safe take-off power to prolong engine life ${ }^{9}$. In general, high levels of accuracy can only be achieved if simulated flight operation is very similar to the underlying flight procedures corresponding to the measured noise levels, i.e. compliance of thrust setting and climb profile (take-off procedures) and configurational setting (approach procedures).

The most prominent example for this kind of tool is the Integrated Noise Module (INM) by the Federal Aviation Administration ${ }^{10}$. INM's implemented standards and methods are recommended by the Society of Automotive Engineers (SAE), see Ref. ${ }^{5}$. The tool is applied by researchers, airport planners, and authorities world-wide to evaluate the impact of airspace management on community noise impact. INM can output maximum or time-integrated noise levels and isocontour areas. With INM, the impact of modifications to flight path, runway/airport layout, and fleet mix on overall ground noise can be accounted for. INM uses Noise-Power-Distance (NPD) tables derived from dedicated flyover noise measurements for each aircraft type to predict ground noise. The NPD data has been recorded along specified, straight horizontal flight segments with constant operational and configurational setting. To predict ground noise impact along a given flight path, it's trajectory has to be assembled from stored and measured straight flight segments. To account for curved flight segments, specific modifications are applied to the noise data. Furthermore, simple approximations are applied to the recorded noise data to account for the actual altitude, flight velocity, and slant distance of the simulated flight. If straight horizontal flight trajectories of infinite length are evaluated, INM will predict precisely the measured ground noise levels for observers located along the flight ground track ${ }^{11}$. Segmentation of a flight procedure into few curved and straight elements can be a decent approximation for common approach trajectories with extensive constant flight operation. Nevertheless, the approximation has its limitations when it comes to more complex airspace routing and flight operations, e.g. airport specific departure routing and advanced low-noise approach procedures. Currently FAA is developing INM's successor the Environmental Design Space (EDS). EDS is based on a more physics based approach in order to study new flight procedures and new vehicle concepts.

At Delft University of Technology INM has been implemented into their trajectory optimization process to study environmental friendly departure procedures for a selected airport scenario ${ }^{12}$. Conventional departure trajectories have been optimized for minimal community noise impact by integrating a geographic information system into the process.

The second example for this group of noise prediction tools is FLULA ${ }^{13}$ by the Swiss Federal Laboratories for Materials Testing and Research. The noise prediction is based on dedicated aircraft noise measurements recorded along individual flyover events with an array of microphones. Polynomial functions are derived from measured spectral noise levels and directivity patterns. Rotational symmetry in noise directivity is assumed hence the polynomials are functions of the polar angle and the distance between aircraft and observer only. Corresponding coefficients are identified and derived from the measurements for each individual aircraft type. To incorporate the influence of aircraft speed, further modifications are applied to the polynomial. Finally, relevant sound propagation effects are accounted for.

A third example, the DLR tool SIMUL ${ }^{14}$, pursues a more physics-based approach compared to the before mentioned tools. In contrast to these tools, SIMUL separates aircraft noise into the contribution of individual components, namely airframe, jet, and fan. Obviously, the dominance of these noise sources is varying along any flight path and will be significantly different along approach and departure procedures. Therefore, separation into these major noise sources seems inevitable for reasonable overall noise prediction

\footnotetext{
${ }^{\mathrm{b}}$ most tools use maximum thrust setting
} 
along arbitrary flight procedures. The indivual noise components are modeled according to knowledge from componential wind tunnel testing and dedicated flyover events. DLR has conducted several flight tests to identify and evaluate dominating noise sources and their directivity corresponding to current speed, thrust, and configurational settings ${ }^{15}$. This large data base allows to further break-down airframe noise into its dominating components, i.e. gear, flap, and slat noise. In conclusion, this approach enables simulation and optimization of realistic approach and departure procedures with complex configurational changes ${ }^{16}$. Noise generating mechanisms can be identified, i.e. different effects of flight speed and operating condition on overall noise emission. For simlicity, rotational symmetry is assumed for the corresponding noise source directivities. Data of preselected and representative flight conditions are stored as input information for the overall noise prediction with SIMUL. Obviously, general application of SIMUL is limited by the aircraft models available in the data base. Currently, the database is limited to one specific aircraft/engine combination. Recent findings from a dedicated flyover noise campaign in 2008 for another aircraft type are yet to be incorporated. Furthermore, the definition of flight procedures is limited by the available data (recorded flight conditions). Again, flight procedures have to be assembled with flight segments corresponding to the available and stored flight conditions.

In conclusion, these tools are ruled out from application within the design process of new aircraft concepts because of their limitation to today's existing technology. In contrast, the focus of the presented work lies on comparative low-noise design studies. To identify design trends and run noise sensitivity studies, it is inevitable to simulate each major noise component with specific individual noise source models. Furthermore, the noise prediction approach has to be more generic and parametric to reflect the effects of geometry modification and operating condition on noise emission. To account for the impact of selected design parameters, e.g. wing sweep and dehidral, on noise emission a more physics-based and complex representation of each component's noise directivity becomes inevitable.

Parametric prediction methodologies form the second group of fast prediction tools. These tools are somewhat empirical but more parametric and physics-based approaches towards overall aircraft noise prediction. Compared to the first group, they represent a good compromise between result accuracy and flexibility towards design modification. Major noise sources are modeled individually with parametrical and semi-emprical noise sources hence effects of geometry and operating condition can be accounted for. The contribution of each modeled noise source will be computed for each one flight position along a given flight procedure. Overall aircraft noise is assembled from all individual noise sources and ultimately emitted from a single point located at the aircraft center of gravity. It is possible to evaluate new aircraft/engine concepts, simulate arbitrary flyover scenarios, and still reflect the basic underlying physical effects. Noise sensitivity studies within the aircraft design phase are enabled and promising design trends can quickly be identified.

The most prominent example of this group of tools is the Aircraft Noise Prediction Program (ANoPP) developed at NASA ${ }^{17}$. Initially, the tool was developed to predict noise for single flyover events. Airframe noise components within ANoPP are modeled with Fink's approach ${ }^{18}$, whereas engine noise is approximated with the methods of Stone ${ }^{19}$ for jet noise and Heidmann ${ }^{20}$ for fan noise. In 2008, two dedicated studies ${ }^{21,22}$ to access NASA's current jet and fan noise prediction capabilites have been published. It was demonstrated that ANoPP's implemented methods for jet and fan noise prediction result in reasonably good overall agreement with the experimental data. Additional engine noise sources can be accounted for such as turbine and core noise. The code is continuously updated and new noise source models are implemented. NASA announced the release of a new version referred to as ANoPP 2.0 beta for the end of $2011^{\mathrm{c}}$. The focus of the new release lies on noise prediction outside of the semi-empirical experience base by applying first principle and multi fidelity approaches. Noise sources are located at their true locations in order to account for interaction effects. More information is scheduled for presentation at upcoming AIAA conferences ${ }^{\mathrm{d}}$. ANoPP was embedded within a low-noise aircraft design framework established at Stanford University. The main objective has been to evaluate the feasibility of such a process accounting for the overall environmental impact. Required aircraft design parameters for the noise prediction with ANOPP are provided by the design modules. Certification noise levels are used as an acoustic constraint within the low-noise design process.

${ }^{\mathrm{c}}$ C.L.Burley, L.V.Lopez: ANOPP2: Progress Update,NASA Spring Acoustics Technical Working Group, presentation, April 21-22 2011, Cleveland, OH

${ }^{\mathrm{d}}$ L.Lopez, C.Burley: Design of the Next Generation Aircraft Noise Prediction Program: ANOPP2, announced for presentation at the 17th AIAA/CEAS Aeroacoustics Conference, Portland, Oregon, June 2011

4 of 24

American Institute of Aeronautics and Astronautics

http://mc.manuscriptcentral.com/aiaa-maa11 
Initial application of their process was presented in $2004^{23}$.

Furthermore, ANOPP has been applied in a joint effort of NASA and Georgia Tech. The overall goal was to evaluate the interaction of multiple engineering disciplines at earlier stages in the aircraft design process. A so-called concurrent approach including ground noise impact has been established in $2006^{24}$. Again, certification noise levels have been used as new design constraints within their process. The focus of the work has been on the overall process and the optimization approach rather than on the noise prediction itself. An extensive number of aircraft and engine design variations has been studied to generate response surface equations for quick overall technology assessment.

A second example is the European tool SOPRANO (Silencer Common Platform for Aircraft Noise calculations). The tool has been developed within the European aircraft noise research program called $S I$ $L E N C E(R)$. ANOTEC consulting developed the tool to provide "a common software platform to assess noise reduction techniques within European projects"; cited from Ref. ${ }^{25}$. The 2007 version models several airframe and engine noise components. The airframe noise is currently modeled with Fink's approach ${ }^{18}$ as well. Major engine noise components are modeled with the SAE methods, Stone's method ${ }^{19}$ (jet noise), and Heidmann's modell ${ }^{20}$ (fan noise). Furthermore, core and turbine noise are accounted for with models found in the literature. Preprocessed or measured source noise data stored in tables is also accepted. Relevant propagation and installation effects are modeled with public domain approaches. The framework is set up to enable future addition of new models. SOPRANO allows the evaluation of individual sources or sum of several components in order to study noise generating effects. The main focus lies on the noise prediction for single flyover events at multiple observer locations. Direct implementation of the code within an aero engine design process was realized 2010 with the TERA2020 (Techno-economic, Environmental and Risk Assessment for 2020) software, a multidisciplinary optimisation tool developed by a consortium of university partners, e.g. Cranfield University ${ }^{26}$.

The third example is ONERA's IESTA (Infrastructure for Evaluating Air Transport Systems) simulation framework $^{27,28}$. Other than the before mentioned tools, IESTA has its focus on multiple flyover events in order to model and simulate current and future air traffic with respect to the environmental impact. Future air traffic is comprised of new vehicles and advanced operational procedures hence requires more physicsbased models. The models for engine noise as implemented in IESTA are similar to the before mentioned methods, i.e. models for jet and fan noise. Predictions by the embedded engine noise models were compared with dedicated experimental data providing reasonably good agreement. Implemented airframe noise sources are modeled according to Fink ${ }^{18}$, except of the slat noise which is simulated with Dobrzynski's approach ${ }^{29}$. For the study of advanced new vehicle designs, airframe noise shielding and interaction effects have to be considered hence are directly incorporated via an ONERA ray tracing method. Overall, IESTA was mainly developed for integration into more complex simulation environments in order to evaluate the overall air transport system.

\section{B. DLR activities}

DLR is investigating its own physics-based noise prediction method in order to (1) fully exploit and incorporate corresponding DLR in-house capabilities, (2) establish DLR-wide accessible noise prediction tool for a wide range of applications, and to (3) provide comprehensible and well-documented solutions for the selected assignments based on a scientific and physics-based methodology. Main assignments for the new tool include investigation of promising low-noise technology, retrofit of existing aircraft, low-noise design of new vehicles, evaluation of noise abatement flight procedures, and simulation of community noise annoyance for selected airport scenarios (multiple flyover events).

To interact with existing DLR software, input and output data require specific translation and correlation among involved disciplines and tools. The tool has to be customized for application at the conceptual aircraft design stage in order to handle the quantity and complexity of available input parameters. Source code access is mandatory for quick modifications in order to generate non-standard and case-specific output data, e.g. level-time-history of a specific noise source frequency spectrum. In general, a flexible and modular tool setup is required to allow for the implementation of interfaces to additional methods and future tools. Different run modes are requested to both incorporate noise prediction into large multidisciplinary tool chains and processes as well as to allow for autarkic and stand-alone application. 


\section{Noise prediction tool and methods}

The Parametric Aircraft Noise Analysis Module (PANAM) is under development by the DLR Institute of Aerodynamics and Flow Technology to address overall aircraft noise prediction at the conceptual aircraft design stage ${ }^{30,31}$. The tool predicts aircraft noise generated during arbitrary approach and take-off flight operations under simultaneous consideration of relevant multidisciplinary effects. Noise generation of an operating aircraft is determined by its design, the relative observer location, configuration setting, and operating condition along the flight path. Furthermore, sound propagation effects under moving conditions have to be applied to transfer static noise emission into ground noise impact due to aircraft flight operation. Individual noise components are modeled with specific semi-empirical and parametrical source models. These models capture major physical effects and correlations yet allow for fast and accurate noise prediction. Individual sources can be monitored and rank-ordered throughout simulated flight operation. If the dominating noise components are identified and modeled it is possible to sufficiently represent the overall aircraft noise emission.

The modular setup of PANAM allows for direct implementation into existing multidisciplinary, conceptual aircraft design codes. If not directly implemented into an aircraft design code, PANAM can be operated in a self-contained mode. In this case, necessary input data have to be provided by the user or come from other DLR tools. Interfaces to various expert tools from other institutes and departments allow to set up case-specific process chains. For example, PANAM can be combined with flight simulation tools to study noise abatement flight procedures. Process implementation of high fidelity tools, e.g. detailed engine cycle analysis, can improve result accuracy. Interfaces to process data from component noise measurements enable the evaluation of these components as if they were installed and operated onboard of an aircraft. For example, the effect of a low-noise gear design could be evaluated along a specific approach procedure.

\section{A. Noise source modelling}

The implemented noise source models are semi-empirical and parametrical. Individual models for selected noise components are available. Each model is derived by computational and experimental data from high fidelity simulations, windtunnel or flyover experiments. The underlying database sets limitations for each model hence general application is constrained. Obviously, result uncertainty increases if models are applied far off the restrictions and conditions within the underlying database. Interactions between individual noise sources are neglected. The noise source models can be separated into two major groups, airframe and engine noise source models. Noise can be evaluated for individual as well as for arbitrary combinations of noise components.

Airframe noise is modeled with DLR in-house noise source models ${ }^{15,29}$. The models are based on data from recent windtunnel and flyover experiments hence reflect the current state of art in airframe design, e.g. sweeped, twisted and tappered wings. Compared to source models from the literature, e.g. Fink's Airframe Noise Prediction Method ${ }^{18}$ as implemented in ANoPP or SOPRANO, the models are defined by a broader parameter set. PANAM now allows for a detailed airframe geometry separation into relevant segments. The wing is separated into multiple acoustic relevant segments with constant flap, slat, and profile geometry. Instead of working with averaged geometry parameters for each kind of noise source, e.g. one representative slat length, multiple noise sources with individual parameter settings are accounted for. This segmented approach enables the noise prediction for unconventional airframe designs, e.g. multiple wing/tailplane configurations and U- or V-tail concepts. Previous and recent findings indicate the importance and dominance of high lift devices on overall airframe noise. Whereas some approaches neglect these devices, the DLR model accounts for both trailing and leading edge devices. Under certain flight conditions flap side edge is assumed to be a dominating airframe noise source ${ }^{1,2}$. Therefore, a new flap side edge noise source model is currently under investigation ${ }^{32,33}$ at DLR and yet to be embedded into PANAM. Sustained research activities towards landing gear noise reduction ${ }^{34,35}$ resulted in a dedicated DLR landing gear noise model. Finally, a simple fully-empirical but parametric model for spoiler noise can be applied if required. All the source models implemented in PANAM are published and open to the public ${ }^{15,29}$.

Engine noise modelling is based on existing models from the literature. The dominating noise sources, i.e. jet and fan noise, are modeled separately. The two standard models for dual-stream jet exhausting from the nozzle $\left(\right.$ Stone $\left.^{19}\right)$ and fan noise $\left(\right.$ Heidmann $^{20}$ ) have been adapted and implemented by the DLR

6 of 24

American Institute of Aeronautics and Astronautics

http://mc.manuscriptcentral.com/aiaa-maa11 
Institute of Propulsion Technology ${ }^{36}$. In comparison to what was presented earlier, some improvements were achieved by modifications of the original fan noise model. Fan noise, i.e. especially the buzz-saw component, has been overpredicted in the past using Heidmann's original model ${ }^{20}$ due to the somewhat antique underlying database. For a better representation of modern turbofan engines with high by-pass ratios, the semi-empirical constants in Heidmann's model have been revisited and updated. Integration of a model to account for the noise absorption due to acoustic liners further improved result accuracy especially under take-off conditions. This model was implemented according to the method described by Moreau, Guérin, and Busse ${ }^{37}$.

The liner damping model is derived from the ray theory. This makes it fast enough for applications in design-to-noise. Configurations with liners mounted in the intake and the bypass duct can be treated. The model works as follows: (1) Applying the method hereafter, the sound field generated by the fan stage is synthetically decomposed into acoustic modes $(m, n)$. (2) The ray structure of the cut-on modes is determined applying a ray theory and assuming hard-wall boundary conditions. (3) Finally, based on the polar and azimuthal angles of propagation ${ }^{\mathrm{e}}$ and the number of bounces along each ray path, the sound attenuation is estimated for each mode; it is assumed that the liners do not significantly modify the propagation angles nor induce mode scattering. The far-field directivity, which is in real applications slightly changed under the effect of the liner, is supposed to remain unchanged. The wall impedance can be predicted using a model based on the geometrical parameters of practical liners and the flow conditions.

For each engine operating point, a modal content has to be synthesized. A specific approach is used for the three noise components identified by Heidmann ${ }^{20}$. For broadband noise, all the cut-on modes are contained and supposed to propagate the same acoustic energy (Equal Modal Energy model). Furthermore, in order to save computing time, the attenuation is calculated only for the centre-frequency of each one-third octave band. The tones at multiple of the blade passing frequency are all assumed to be generated by the rotor-stator interaction mechanism. The azimuthal order of those modes is calculated by applying the Tyler and Sofrin rule $(m=h B-k V)^{38}$. The same power amplitude is given to all the radial orders. Finally, the modal content of buzz-saw noise has to be determined in transonic regime of the fan. Since buzz-saw is a rotor-locked mechanism, the azimuthal order of each engine order tone can be assumed to have the same value as the engine order itself. Then, within a given one-third octave band all the engine orders are considered to have the same amplitude. The energy of the radial order components is chosen to be equal too.

The implementation of a liner model makes it possible to investigate the influence of the liner length. A long intake and bypass-duct equipped with damping material could offer a feasible solution to reduce the noise contribution from the fan.

\section{B. Input parameters}

Input data requirements for the implemented noise source models include (1) aircraft design, (2) engine design parameters with a detailed engine performance deck, (3) a simulated flight trajectory with configuration settings and operating conditions, and (4) the location of the observer. Relevant input data can be provided through corresponding tools or has to be generated by the user in order to initiate the overall noise prediction process. Theoretically, the required parameters can be generated with available and standard conceptual aircraft design systems.

PANAM was developed for direct application within the aircraft design synthesis code PrADO ${ }^{39}$, an inhouse development of the Technical University Braunschweig, Germany. PrADO can assess the feasibility of new aircraft concepts at the conceptual aircraft design stage. The PrADO framework is comprised of individual design modules each dedicated to certain tasks or disciplines. These design modules run interactively until predefined design requirements are meet. PANAM can be operated as one of these self-contained modules within the framework hence can directly access the automatically generated input parameters.

Furthermore, PANAM can be operated within DLR's TIVA ${ }^{40}$ simulation environment. DLR has established a common system to enable distributed multidisciplinary conceptual aircraft design ${ }^{41}$. The TIVA system is based upon a common data exchange format to establish a framework ${ }^{42}$ for the integration of tools and methods from multiple disciplines and experts. Currently, various tools from specific disciplines are available

eangles at which the rays impinge on the wall 
and can be implemented into individual process chains to provide the required input data for the noise prediction. The generated data of each implemented tool is collected and stored in the standardized data format. Ultimately, the data can be made accessible for the noise prediction via network connection.

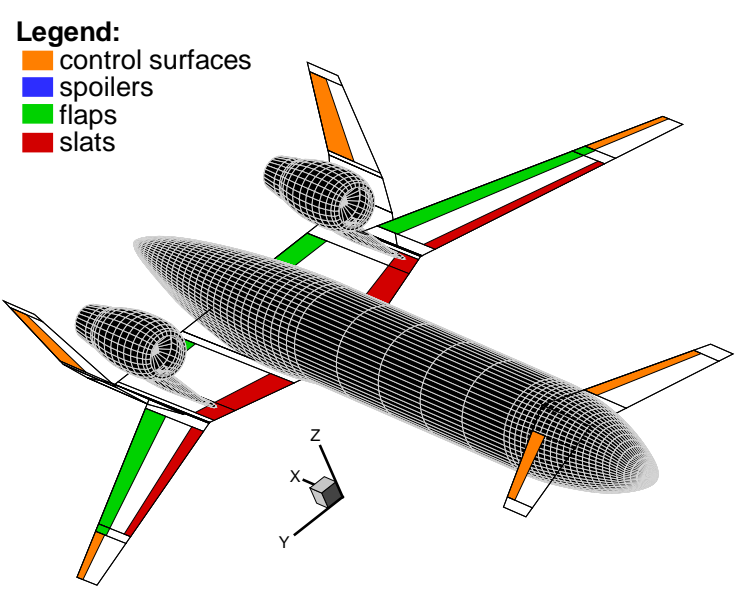

Figure 1. Automated airframe geometry segmentation into acoustic relevant elements (here: DLR Low-Noise Aircraft)
(1) Aircraft design parameters for each relevant and modeled component are required. Optionally, the wing geometry can be separated into relevant segments with constant shape and high lift design. If dealing with complex configurations, the segmentation approach becomes inevitable to guarantee feasible representation of the geometry. The noise prediction algorithms have been modified to optionally account for such a segmented geometry input. If the user has to provide the required input parameters, it is still possible to switch back to the simplified input format, i.e. each relevant component group is reduced to a corresponding, representative, and averaged geometry element. PrADO has been updated to automatically provide a detailed geometry segmentation. A newly implemented algorithm identifies the acoustical relevant component segments and automatically generates a detailed input parameter set for PANAM, see Fig. 1. Depending on the selected design tools, the TIVA framework will provide either averaged component or detailed segmential parameters.

Optionally, possible noise shielding effects due to aircraft geometry and relative engine location can be simulated within the TIVA framework. Direct coupling of the aircraft design code PrADO and PANAM to the DLR ray tracing tool SHADOW ${ }^{43}$ allows for automated processing and evaluation ${ }^{44}$ of these effects, see Fig. 1.

(2) Engine design parameters and performance decks have to be generated through dedicated thermodynamic engine cycle modelling. Detailed input parameters are crucial for the result accuracy of engine noise prediction. If PANAM is embedded into a multidisciplinary process with corresponding engine tools, i.e. DLR's VarCycle ${ }^{45}$ tool $^{\mathrm{f}}$, these parameters are automatically generated and provided as input. Embedded into a conceptual aircraft design tool such as PrADO, certain detailed engine parameters cannot be generated due to their complexity, e.g. rotor and stator blade count. In this case, the detailed engine parameters have to be manually evaluated and integrated into the overall process. If the focus is airframe design, i.e. working with one specific engine type or concept, the detailed engine geometry parameters are pre-evaluated once and then assumed constant during further engine scaling within predefined limitations.

(3) Flight trajectories are discretized into individual flight positions. For each flight position the aircraft configurational setting, operating condition, and the location of the aircraft are held constant for the duration between two successive flight positions. The required flight trajectory data for PANAM includes aircraft and engine operating conditions, configurational setting, and aircraft orientation and location.

The trajectory data can automatically be generated within the PrADO framework or with any available flight simulation tool in the TIVA environment, e.g. MisSim ${ }^{46,47}$. Furthermore, actually recorded flight data and radar data can be transformed into the required input format for further analysis if required.

(4) Arbitrary observer locations can be selected for the noise prediction. Depending on the selected task, e.g. evaluation of level-time-histories or isocontour areas, both individual and arrays of observers can

\footnotetext{
${ }^{f}$ VarCycle is an one-dimensional, off-design performance calculation program developed by the Institute of Propulsion Technology (AT)
} 
be processed. Individual observer property settings can be defined for each location, i.e. ground resistivity to air, observer height, and population density if required. This allows to define and simulate arbitrary airport surroundings with a realistic population distribution only limited by the data availability ${ }^{\mathrm{g}}$.

\section{Noise prediction}

Each flight operation is simulated by quasi-stationary flight segments. During each flight segment the aircraft location/orientation, operating condition, and configuration of the aircraft are kept constant hence noise emission is assumed to stay constant as well. Obviously, the separation into these constant flight segments determines result accuracy. For noise prediction with PANAM a maximum segment flight time of 0.5 seconds has been identified, i.e. a minimum of two quasy-stationary flight positions per second of flight time are required. The spacial distance between two quasi-stationary flight situations depends on the flight speed and is obviously not constant.

For each combination of quasi-stationary flight position and observer location, the farfield sound pressure level frequency spectrum is computed ${ }^{\mathrm{h}}$, audible range: $20-20 \mathrm{kHz}$. Frequency dependent sound propagation effects are applied to transfer static noise emission into ground noise impact due to aircraft flight operation. Sound propagation effects include geometrical spreading, ground attenuation, convection effects, and atmospheric absorption. Sofar, wind and temperature effects have not been in the scope of the presented research activities hence are not accounted for. Common psycho-acoustic weighting functions can be applied to the spectral data for better simulation of human sound perception. Finally, the predicted frequency spectra is translated into corresponding single-event-levels, e.g. A-weighted Sound Pressure Level $\left(\mathrm{SPL}_{A}\right)$ or Tone-corrected Perceived Noise Level (PNLT). The predicted time-history of the single-event-levels can be integrated to obtain common flyover noise metrics such as the Effective Perceived Noise Level (EPNL).

\section{Standard output data}

Individual flyover events are the default application for PANAM. The data output includes noise levels for each individually modeled aircraft component as well as levels for the overall aircraft. Noise level frequency spectra for individual components can be provided for any specified operating condition along a simulated flight path. Furthermore, level-time-histories, weighted or time-integrated noise metrices can be predicted for each flyover event. The time-integrated and weighted output data includes the standard flyover noise measures, e.g. (A-weighted) Sound Pressure Level and Effective Perceived Noise Level (EPNL). For a grid of observer locations the distribution of noise levels as received on the ground can be generated. Animated noise footprints for each one time step as well as visualizations of time-integrated noise footprints can be generated. This allows for real-time evaluation of the influence of aircraft operating conditions and configuratinal settings on the overall noise radiation. Noise related effects can be identified, visualized, and ultimately analyzed ${ }^{48}$.

Isocontour areas of the max. Sound Pressure Level (SPL) can be translated into a probability distribution of aircraft noise induced awakenings ${ }^{49}$. Multiplied with a given population density, one can evaluate the number of affected people, i.e. awake people due to a single aircraft flyover noise event. This scalar value is well suited as a design objective within an optimization process. Obviously, the population density around a typical airport is very inhomogeneous. But due to the complexity of modelling the communities around a real airport and the lack of available data, usually an average and constant population density is assumed ${ }^{\mathrm{i}}$.

Multiple flyover events can be simulated with the code as well. Arbitrary combinations of individual flyover events are arranged into a multiple flyover scenario. Equivalent Sound Pressure Levels (aequivalenter Dauerschallpegel, $L_{e q}$ ) are computed for arbitrary combinations of aircraft, fleet mix, flight operation, and runway layout. The computations are performed according to Ref. ${ }^{50}$.

$$
L_{e q}=k \cdot \log 10\left(\frac{t_{r e f}}{T} \cdot \sum_{i=1}^{N} g_{i} \cdot 10^{L_{E, i} / k}\right)+C
$$

The flyover events $(N)$ within a predefined time span $(T)$ are summed up for an arbitrary observer location. $L_{E, i}$ is the noise level for one individual flyover event $(i), t_{r e f}$ is the specific time span defined for $L_{E}, g_{i}$ is

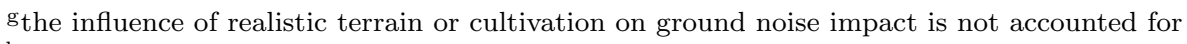

$\mathrm{h}_{1} / 3$-octave bands

${ }^{\mathrm{i}}$ current population density of Germany: 231 people per square kilometer
} 
the time dependent weighting factor. Parameter $k$ depends on the summation of the individual noise levels, e.g. $k=10$ for energetic summation. Finally, the resulting levels are scaled/normalized by factor $C$. The most common noise metrices can be evaluated according to the parameter setting in Table 3 .

To speed up computational time, the ground noise distribution for each combination of (1) aircraft, (2) flight procedure, and (3) selected runway is pre-computed and stored within a database. Simulating a selected flight plan, comprised of arbitrary combinations of available settings (1) to (3), is then predicted based on the pre-computed database within seconds.

The most commonly used $L_{e q}$ is the Day-Evening-Night Sound Level $\left(L_{D E N}\right)$. A noise response relationship by the European Commission translates the $L_{D E N}$ into aircraft noise induced annoyance ${ }^{51}$. Ultimately, $L_{D E N}$ isocontour areas for multiple flyover events/scenarios can be reduced to one scalar value applicable as a design objective within an optimization process.

\section{E. Optional output data}

Geographical noise impact visualisation enables a better understanding on how aviation impacts local communities and their annoyance towards noise pollution. Overlapping noise isocontour plots with maps of local communities will indentify noise affected areas and population. Hereby, noise impact on sensitive areas such as highly populated residential areas can directly be evaluated. For a predefined airport scenario with given runway layout and airspace routing, PANAM transforms the flight trajectories and resulting noise isocontour areas into WGS84 coordinates to load them into a geographical visualisation tool, e.g. Google $\operatorname{Earth}^{52}$. Various noise metrics for individual or multiple flyover events can be visualized.

Gaseous engine emissions are summed up along simulated flight procedures. Obviously, this optional output depends on the availability of emission data for the selected engine. If emission data is available, flight procedures can be modified for best environmental performance, i.e. evaluation of noise vs. gaseous emissions vs. required fuel. Results of an initial trade study for individual flyover events have been published in $2009^{48}$.

\section{Comparison with experimental data}

Each implemented noise source model has separately been validated by comparison with componential windtunnel and other experimental data ${ }^{15,29,36}$, i.e. data recorded under steady laboratory conditions. To evaluate the interaction of these individual noise sources under consideration of source movement and sound propagation effects, predicted overall aircraft ground noise is compared with existing ground noise measurements. If working with measured flyover noise levels one has to keep in mind that aircraft noise measurements in a realistic environment are subject to inherent imprecision and limited replicability ${ }^{7}$, e.g. due to varying atmospheric conditions. Therefore, good overall agreement of predicted noise levels with actual measurements becomes very difficult and can only be achieved to a certain extend, especially if only simplified sound propagation effects are accounted for. Nevertheless, only a dedicated comparison of simulated versus measured aircraft ground noise levels can indicate feasibility and quality of any applied prediction methodology.

Three recent DLR flyover noise campaigns are selected for the comparison: (1) Parchim 2006 campaign, A319 with CFM56-5A5 engines, October 2006, (2) Braunschweig 2009 campaign, VFW-614 ATTAS with Rolls-Royce/SNECMA M 45 H Mk. 501 engines, June 2009, and (3) Braunschweig 2010 campaign, B737-700 NG with CFM56-7B26 engines, September 2010. The aircraft geometries are re-designed with PrADO ${ }^{39}$ and relevant engine design parameters and performance data come from DLR's VarCycle ${ }^{45}$ tool. Flight operation of these aircraft is simulated according to recorded flight test data in order to predict ground noise impact at the selected observer locations.

(1) An A319 was operated along 9 approach and 9 departure procedures. Ground noise measurements have been recorded at 12 departure and 13 approach observer locations with both ground and $1.2 \mathrm{~m}$ microphones. Initial comparison for this campaign has been presented earlier ${ }^{30}$. Recent modifications to airframe and engine noise source models and the implementation of an acoustic lining model further improved the overall agreement of prediction and experiment. Figs. 4 to 6 show results for selected approach and depar- 
ture trajectories of the campaign. The procedures have been selected according to the applications within this paper. Absolute SPL values as well as SPL variation in time is reasonably well predicted along all three procedures. SEL noise levels are presented to compare measured and predicted noise energy impact. Most predicted SEL levels are in good agreement to the measurements, i.e. within a $\pm 2 \mathrm{~dB}$ margin to measured SEL levels. Along the LDLP procedures, ground noise is significantly overpredicted at locations 1 and 5, i.e. the most distant locations. In general, PANAM slightly overpredicts ground noise levels for the A319 especially for distant observer locations. This can be adressed to a combination of still existing (but reduced) engine noise overprediction and the fact that weather effects are not taken into account. Take-off noise prediction has been significantly improved due to the modifications compared to results presented in Ref. ${ }^{30}$.

(2) In 2009, DLR's flying testbed ATTAS was operated along different approach trajectories. New radical and unconventional approach procedures have been evaluated during this test campaign. Inspired by initial concepts and flight tests by $\mathrm{NASA}^{58}$ a new procedure was developed and flight tested, the Helical Noise Abatement Procedure (HeNAP). Furthermore, a steep approach and a conventional approach procedure have been integrated into the flight test schedule. Initial comparison of experimental and computational noise levels have been presented in Ref. ${ }^{48}$.

Fig. 7 shows the ground flight tracks for all 7 test flights along with the observer ground locations. In the past, absolute levels indicated significant discrepancies between experiment and prediction, especially if noise shielding effects have been accounted for within the simulation. This effect was mainly adressed to adverse weather conditions corrupting the measurement of complex noise shielding effects. Futhermore, simulation of the unique aircraft and engine design of the ATTAS is complex and error-prone. Nevertheless, noise dislocation effects and comparative level differences were in good aggreement.

Modifications to the engine noise source models and the application of noise shielding effects to only the forward fan noise improved the overall agreement to the measurements. Yet, discrepancies in absolute ground noise levels are still existent. Figs. 9(a) and 9(b) show the time-level-histories along three different approach procedures at two selected observer locations. Fig. 8 shows componential noise evaluation at observer location 4 subject to engine idle noise along steep approach procedures. Former strong underestimation of the engine idle noise is reduced. Fig. 9(c) shows prediction results along the curved flight segments.

(3) In 2010, DLR operated a Boeing 737-700 along different approach procedures to investigate advanced airspace re-routing schemes for Frankfurt International Airport. To assess the prediction capabilities for this type of aircraft, recorded noise level-time-histories are compared to initial predictions with PANAM. The presented results are preliminary and still under investigation. The general noise generating mechanisms, i.e. the high lift system and the landing gear for airframe noise, are assumed to be consistent with the PANAM noise source models. A detailed engine design for this aircraft is still under investigation hence a simplified engine model had to be applied. Fig. 10 shows the predicted and measured SPL(A) time-histories and the SEL at two selected observers directly located under the common approach path. Two direct flyover events have been selected for this initial comparison. Both flights pass the observer locations on similar flight altitudes but with different configurational setting. Along flight 1, the landing gear is extracted right after flyover of observer 1 . The aircraft continues with extracted landing gear and passes observer location 2, hence the level differences between the observers can be adressed to landing gear noise contribution. Along flight 2, landing gear is extracted prior to reaching the observers causing increased noise levels. Overall, the predicted levels and time-histories of both flights are in satisfying agreement with the measurements. A more detailed analysis of these preliminary results is required in order to verify the initial assumptions.

\section{Application}

\section{A. Flight trajectory optimization}

PANAM is coupled with the DLR Mission Simulation (MisSim) tool ${ }^{46,47}$ to enable the environmental analysis and optimization of flight procedures. A dedicated and automated process is established within DLR's TIVA $^{40}$ simulation environment. MisSim automatically generates a flight dynamics model for the given aircraft configuration and then simulates a prescribed mission, flight manoeuver, or prepares the model for other forms of analysis, e.g. linearization for handling quality evaluations. Missions may be simulated in two ways: (1) quasi-statically by using an automatically generated inverse point mass model (inputs are the

11 of 24

American Institute of Aeronautics and Astronautics

http://mc.manuscriptcentral.com/aiaa-maa11 
flight path and speed profiles, outputs are engine and controls settings, etc.) or (2) by means of a point mass or full 6 DOF model in combination with a generic autopilot. The latter variant has become the preferred option being considerably more flexible, especially when thrust limits are reached.

A parameter study for take-off and climb-out profiles is simulated and evaluated for noise and gaseous emission. The reference aircraft (A319 type) takes off, reduces power to $90 \%$ and accelerates to a calibrated airspeed of $210 \mathrm{kts}$. This profile is varied in order to investigate the environmental impact:

(1) modified thrust setting with constant airspeed (CAS): thrust reduction to $80 \%$ (resulting in a lower climb rate but less engine noise) or increase to $100 \%$ (resulting in a higher climb rate but more engine noise)

(2) modified airspeed with constant thrust setting: thrust remains $90 \%$ but airspeed is increased to $220 \mathrm{kts}$ (resulting in a lower climb rate) or decreased to $200 \mathrm{kts}$ (resulting in a higher climb rate)

Noise prediction results are depicted in Fig. 11 and gaseous engine emissions are listed in Tab. 1. Engine emissions are summed up over a $20 \mathrm{~km}$ segment along each simulated departure procedure.

The Effective Perceived Noise Level is evaluated in order to account for both absolute noise level and time duration of each flyover event. Fig. 11 shows the EPNL values along the ground flight tracks. Furthermore, altitude, airspeed (TAS), and thrust setting profiles are presented for each flight. Noise relevant effects with respect to these profiles can directly be identified in the EPNL data. Reduction of thrust along with constant TAS requirements directly results in reduced flight altitudes as depicted in Fig. 11(a). Obviously, reduced ground distance counteracts reduced engine noise emission due to lower thrust setting, hence ground noise levels are increased. With respect to ground noise impact, further thrust increase to $100 \%$ would be benefitial. But as can be expected, thrust increase results in higher emissions compared to the flight with lower thrust settings, see Table 1 . Therefore, a thrust setting of $90 \%$ is the predicted optimum.

Fig. 11(b) shows the results for the airspeed variation with constant thrust setting. It might seem surprising that the higher airspeeds are not correlated with increased gaseous emissions according to the data. This can be traced back to the reduction in flight time along the selected flight segment compared to flights with lower airspeeds. Higher airspeeds with constant thrust setting result in lower flight altitudes hence increased ground noise levels. Limiting the airspeed (CAS) to $200 \mathrm{~m} / \mathrm{s}$ provides more engine thrust to build up flight altitude hence reducing ground noise. A 2 EPNdB noise reduction is predicted early along the flight path but decreases downwards the flight trajectory. With only small increase in gaseous emissions, a velocity reduction down to $200 \mathrm{~m} / \mathrm{s}$ along with constant thrust setting is the predicted optimum.

\begin{tabular}{|l|c|c|c|c|c|c|c|c|}
\hline mod. & flight time [\%] & fuel [\%] & $\mathrm{H}_{2} \mathrm{O}[\%]$ & $\mathrm{CO}_{2}[\%]$ & $\mathrm{NO}_{x}[\%]$ & $\mathrm{CO}[\%]$ & $\mathrm{HC}[\%]$ & soot [\%] \\
\hline $\mathrm{CAS}+10 \mathrm{~m} / \mathrm{s}$ & -2.79 & -1.93 & -1.93 & -1.93 & -1.75 & -1.93 & -1.91 & -0.78 \\
$\mathrm{CAS}-10 \mathrm{~m} / \mathrm{s}$ & +3.29 & +2.29 & +2.29 & +2.29 & +2.09 & +2.29 & +2.26 & +1.10 \\
\hline thrust $+10 \%$ & -0.51 & +6.27 & +6.27 & +6.27 & +5.98 & +6.27 & +6.27 & +4.19 \\
thrust $-10 \%$ & +0.25 & -6.29 & -6.29 & -6.29 & -4.06 & -6.29 & -6.27 & -3.03 \\
\hline
\end{tabular}

Table 1. Gaseous emissions along departure procedures up to a distance of $20 \mathrm{~km}$ to the airport: delta with respect to reference $(\mathrm{CAS}=210 \mathrm{~m} / \mathrm{s}$, thrust $=90 \%)$

\section{B. Low-noise aircraft design}

Significant contribution to overall ground noise for approaching aircraft can be traced back to the leading edge devices (LEDs). Rank-ordering the airframe noise sources during approach operation identifies conventional LEDs as one predominating noise source until extraction of the landing gear ${ }^{2}$. DLR is investigating new advanced high-lift LEDs in order to reduce componential noise emission without any decrease in vehicle flight performance. To evaluate the theoretically achievable noise reduction potential of such advanced LED concepts, a simple trade study has been performed. Low-noise LED have been installed on-board of a conventional, medium-range transportation aircraft ${ }^{j}$ in order to predict the influence on overall ground noise impact compared to the reference design. It is assumed that an optimal low-noise LED design can be identified and is installed, i.e. LED noise emission can be significantly reduced but overall aircraft flight performance remains constant. Such an optimal design allows to simulate both reference and low-noise vehicle along identical flight paths since overall aircraft flight performance is not modified or affected by the new LEDs. The dominating noise contribution of standard LEDs along simulated approach flight operation

j150 PAX, design mission: $4800 \mathrm{~km}$, cruise mach 0.78, cruise alt 8500 - $12500 \mathrm{~m}$ 
is simply assumed to be eliminated for the new design, i.e. the LED noise emission is reduced significantly with respect to remaining airframe noise components.

The area subject to potential noise reduction due to advanced LEDs can be determined according to the configurational setting along the flight path, e.g. an approach procedure with late gear extraction seems to be most advantageous. Such a procedure is the Continous Descent Approach with Late Gear Extension (LCDA) under investigation by DLR ${ }^{16}$. According to Ref. ${ }^{53}$ there is no intermediate flight segment but a continous descending flight path. Moderate descent angles will allow for simultaneous altitude and speed reduction along the descent. This results in a higher flight path along with reduced flight speed which is both benefitial to overall aircraft noise reduction. Early configurational changes become necessary to guarantee required deceleration of the aircraft along the continuous descent. Engines can be operated on low thrust settings along most of the procedure resulting in significantly reduced engine emission. Therefore, airframe noise contribution is of high importance and dominance along such a flight procedure. Late gear extension within prescribed safety margins allows for additional reduction of overall aircraft ground noise impact but usually results in wide-spread dominance of LED noise. Noise source dominance along a LCDA is depicted in Fig. 12(a). The area of dominating LED noise contribution along this procedure is highlighted. If LED noise could be significantly reduced, overall ground noise levels in this area are minimized. Additional landing gear noise reduction would furthermore reduce ground noise pollution. Predicted max. SPL along the ground flight track for the reference aircraft are compared to noise levels for an vehicle with low-noise LED, low-noise gear, and low-noise LED \& gear, see Fig. 12(b) indicating a significant overall ground noise reduction.

\section{Community noise annoyance}

A quick way to identify possible impact of aircraft noise on local communities is to correlate predicted noise isocontour areas with geographical information. An example application with Google Earth ${ }^{52}$ is presented. A conventional medium-range transportation aircraft is simulated along a LDLP approach towards runway $28 \mathrm{~L}$ of Portland International Airport. For comparison, an aircraft with low-noise LED is operated along a LCDA procedure. The predicted results can directly be mapped onto the airport surroundings as depicted in Figs. 13(a) and 13(b) respectively. The max. SPL isocontours clearly indicate the difference in noise distribution and ground noise impact on local communities along both procedures. The dominating influence of conventional LED on overall aircraft ground noise is eliminated and ground noise levels along the LCDA are furthermore reduced, as depicted in Fig. 13(b). Max. SPL and EPNL noise level are predicted at the permanent noise measurement sites ${ }^{k}$ installed around the airport. Compared with the LDLP approach, community noise levels around "Lake" are significantly reduced along the LCDA. Application of the lownoise leading edge device further reduces noise pollution along the approach path, i.e. at measurement site "McGill".

\begin{tabular}{|l|c|c|c|c|}
\hline modification & metric & P04 "Argay" & P05 "Lake" & P06 "McGill" \\
\hline LCDA procedure & EPNL [EPNdB] & -2.3 & -5.8 & -0.1 \\
& max. SPL(A) [dBA] & +0.2 & -3.3 & +0.0 \\
\hline \multirow{2}{*}{ SLED \& LCDA procedure } & EPNL [EPNdB] & -2.5 & -6.6 & -4.1 \\
& max. SPL(A) [dBA] & +0.2 & -3.3 & -5.8 \\
\hline
\end{tabular}

Table 2. Max. SPL and EPNL level differences wrt reference a/c along LDLP (permanent noise measurement sites)

A large variety of noise metrics is available to further quantify community noise annoyance. Most of these noise metrics are highly empirical and require population data as an input. The Aircraft Noise Induced Awakenings ${ }^{49}$ directly correlate simulated flight operation with the number of awakened people around an airport. This scalar number can then be used as an objective within an automated optimization process. Not having access to any population data in a feasible resolution or format, an interface to read and modify CORINE land cover data ${ }^{54}$ has been implemented. The CORINE data does only indicate the land usage but does not provide any number of people. In a first step towards more realistic simulation of selected airports and their surrounding, residential areas are identified in the CORINE data in order to only account for populated areas. Fig. 14 shows the populated areas around Frankfurt airport.

kaccording to http://www.portofportland.com 
The probability of aircraft noise induced awakenings is evaluated within a selected subsection of the Frankfurt area. The selected area is 15 by $20 \mathrm{~km}$ large and located north-east of the airport. The community noise is simulated for a generic and a some-what more realistic population distribution ${ }^{1}$. It is assumed that approximately 280000 people live in this selected area, i.e. an average of 760 people per square kilometer for the homogeneous population density. Two scenarios are evaluated and compared: reference aircraft along LDLP approach and low-noise vehicle (LED) along SCDA procedure. In both cases the max. SPL(A) isocontour areas are translated into the probability of aircraft noise induced awakenings ${ }^{49}$, see Fig. 15(a). Multiplying this probability with the corresponding population density provides the actual number of aircraft noise induced awakenings. Consideration of a homogeneous population density results in a significantly different distribution of local awakenings compared to a more realistic population scenario as depicted in Figs. 15(b) and 15(c). Not only the location but also the total numbers of awakenings are significantly different. Both population distributions result in approximately $15 \%$ less aircraft noise induced awakenings due to operation of the low-noise aircraft ${ }^{\mathrm{m}}$. Further and more detailed distribution of the people would significantly influence and change predicted percentages of awakenings. Accounting for a some-what more realistic population density can dominate the outcome of the simulation hence feasible evaluation of community noise impact should allways incorporate realistic population densities.

\section{Airspace and airport simulation}

An automated process chain for airspace and airport simulation with integrated noise prediction capabilities ${ }^{55}$ is under development at DLR using FAA's SIMMOD ${ }^{56}$ tool. Different air traffic scenarios can be defined and evaluated for their impact on capacity and delays. The scenarios are made up of the airport/runway layout, the airspace segmentation, and the corresponding flight path alignment. Individual aircraft movements are simulated along airspace routes, runways, taxiways, and gates according to a predefined schedule. Ultimately, the combination of all scenario parameters will determine the overall airport and airspace capacity.

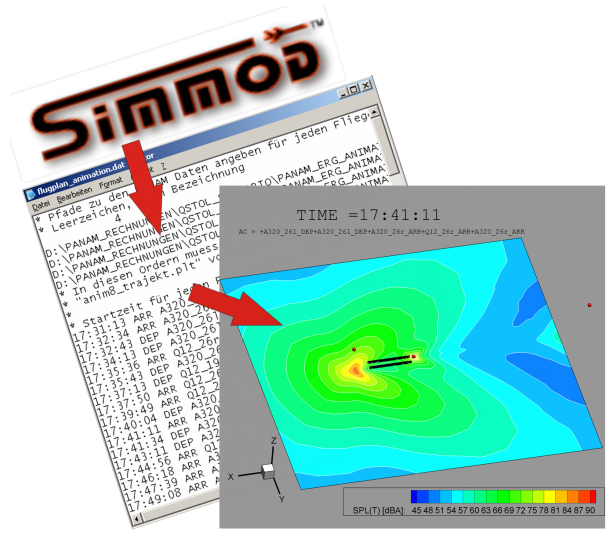

Figure 2. SIMMOD airspace simulation with integrated noise prediction capabilities PANAM can read and translate the data format of SIMMOD to evaluate overall ground noise impact. Obviously, the predicted scenario noise levels depend on (1) the individual aircraft type, (2) the runway layout, (3) the airspace routing/flight procedure, and (4) the flight schedule. With respect to noise prediction, one major simplification is applied to reduce computational costs to a minimum. The ground noise levels are assumed constant for each specific combination of (1) - (3). In other words, each flight of one specific aircraft type along one specific flight path towards one specific runway is assumed to result in identical ground noise levels. Consequently, corresponding noise data is only predicted once in order to generate an extensive data base for further access. For any given and consistent scenario, the stored noise data can be assembled according to the underlying flight schedule and by applying time dependent weighting factors as listed in Table 3. Ultimately, an airport and airspace scenario can simultaneously be evaluated for community noise and air traffic delays in a very short time, see Fig. 2. A second runmode has been implemented into PANAM to quickly identify noise related trends without simulating the entire airspace and airport. For a given SIMMOD scenario, i.e. fixed combination of (1)-(4), selected and individual flight movements are manually replaced by new aircraft and flight procedures under the assumption that capacity issues remain constant. This enables to quickly evaluate new technology with respect to an overall airport community noise impact, e.g. by replacing a specific aircraft type with a modified, low-noise replacement with similar flight performance.

A generic two runway airport layout with 719 flight operations of one representative medium sized transportation aircraft is simulated. Fig. 16 shows the predicted Day-Evening-Night Sound Level (LDEN) for an average day. To study the impact of low-noise leading edge technology on longterm noise pollution of the airport surrounding, fifty percent of all reference flights are replaced by the silent LED vehicle assuming

\footnotetext{
${ }^{1} 280000$ people are equally distributed only within the CORINE residential areas

$\mathrm{m}$ predicted percentages are identical because of the assumption that selected residential areas have a constant population density as well
} 
identical flight performance and airspace routing. Furthermore, all approaching aircraft are simulated along LCDA procedures. These modifications show significant community noise reduction in the vicinity of the scenario airport, i.e. due to the new approach and due to the low-noise LED technology. Along the common approach path, LDEN levels are reduced by 2-3 dB if the low-noise LED design is realized on-board of approximately $50 \%$ of the simulated vehicles and if all aircraft operate along LCDAs.

\section{Conclusion \& summary}

The overall aircraft noise prediction tool PANAM is presented. Prediction results are compared to existing ground noise measurements for three aircraft along multiple flight procedures, i.e. departure and approach. Dedicated comparison of simulated and measured noise levels and noise dislocation effects indicates feasible overall aircraft noise prediction capabilites. Overall, the quality of the presented noise prediction methodology can be ranked as well suitable to support decision making in the context of aviation noise and emission pollution. Result reliability is reduced if existing noise source models are applied towards vehicle concepts far off the design space specified by the underlying empirical data. Yet, if general noise generating mechanisms are kept consistent with the empirical data base, i.e. fixed design principles for individual noise sources, reasonable and reliable results can be achieved. Comprehensive results can be provided in order to enable comparative low-noise vehicle design analysis.

PANAM can be operated within the aircraft design synthesis framework PrADO or the DLR framework TIVA to enable fully automated aircraft design trade studies. Promising low-noise technologies can be evaluated at early design stages in order to influence the overall vehicle design. PANAM can be applied to investigate the environmental evaluation of flight operations. Individual flights as well as multiple flyover scenarios can be evaluated. An interface to a fast time airspace simulation environment allows to investigate case-specific airport scenarios and study the environmental impact. Geographical noise mapping and processing of CORINE land cover data is implemented in order to evaluate community noise impact. It is demonstrated that feasible evaluation of community noise metrics should incorporate realistic population densities.

Future work will include implementation of new noise source models and further data comparison with existing flyover noise measurements. In the near future, airframe noise prediction capabilities will be enhanced by a flap side edge noise mode ${ }^{32,33}$. Recent development of an open-rotor noise prediction program belongs to ongoing engine noise activities at DLR. The selected acoustic model is based on the analytical solution proposed by Hanson ${ }^{59}$. This separate program can be linked to PANAM via the TIVA simulation environment ${ }^{40,41}$ in order to enable overall aircraft noise prediction for open-rotor vehicles.

Finally, detailed evaluation of PANAM's existing real-time noise prediction capabilities in the context of real-time simulation environments is planed for the future. For example, noise prediction capabilities could be integrated into air traffic management software or into ground-based flight simulators to increase the pilot's community noise awareness.

\section{Acknowledgments}

The assistance of Dr. Wolfgang Heinze ${ }^{\mathrm{n}}$ towards a low-noise aircraft design process and his continous support is greatly appreciated. The authors thank Dr. Werner Dobrzynski ${ }^{\circ}$ for his support and expertise in the area of noise source modelling. The authors would like to thank Mr. Manfred Keil ${ }^{\mathrm{p}}$ for his support towards applying the CORINE data. Furthermore, the authors thank Mr. Oliver Schneider ${ }^{\mathrm{q}}$ for his support towards a noise prediction interface to SIMMOD. The authors thank Mr. Helmut Toebben ${ }^{q}$ and Mr. Vilmar Mollwitz $^{\mathrm{q}}$ for the B737 flight data. Furthermore, the authors thank Mr. Tom Otten ${ }^{\mathrm{r}}$ for his assistance in modelling the thermodynamic engine cycle hence providing high quality engine performance decks.

\footnotetext{
${ }^{\mathrm{n}}$ Institute of Aircraft Design and Lightweight Structures, Technical University of Braunschweig, Germany

${ }^{\circ}$ DLR, Institute of Aerodynamics and Flow Technology

PDLR, Earth Observation Center (EOC)

qDLR, Institute of Flight Guidance

${ }^{\mathrm{r}} \mathrm{DLR}$, Institute of Propulsion
} 


\begin{tabular}{|c||c|c|c|c|c|c|c|}
\hline \multicolumn{1}{|c|}{$L_{\text {eq, gew }}$} & $L_{E, i}$ & $k$ & $C$ & \multicolumn{4}{|c|}{ Time dependent weighting $g_{i}$} \\
& & & & $0600-0700$ & $0700-1900$ & $1900-2200$ & $2200-0600$ \\
\hline$L_{\text {eq(4) }}-A$ & $L_{A Z}$ & 13.3 & 0 & 1.5 & 1.5 & 1.5 & 0 \\
$L_{\text {eq(4) }}-B$ & $L_{A Z}$ & 13.3 & 0 & 1 & 1 & 1 & 5 \\
$L_{D N}$ & $L_{A X}$ & 10 & 0 & 10 & 1 & 1 & 10 \\
$L_{D E N}$ & $L_{A X}$ & 10 & 0 & 10 & 1 & 3.162 & 10 \\
$N E F$ & $E P N L$ & 10 & -48.63 & 16.67 & 1 & 1 & 16.67 \\
\hline
\end{tabular}

Table 3. Equivalent sound pressure level $L_{e q}$

\section{Figures}

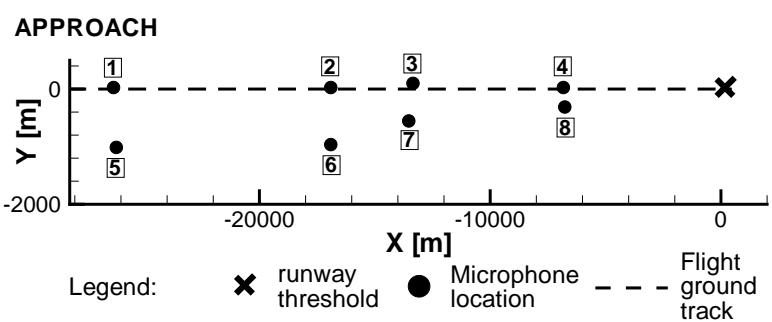

(a) approach

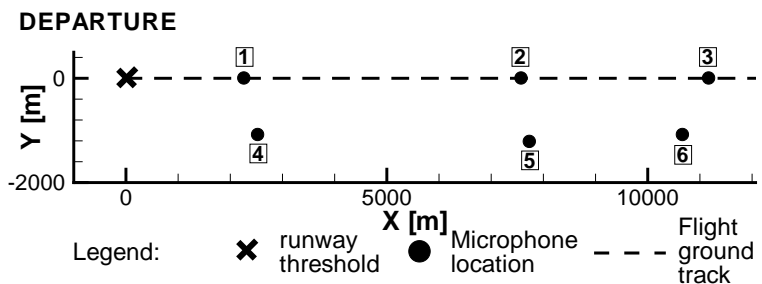

(b) departure

Figure 3. Parchim Campaign: Observer locations

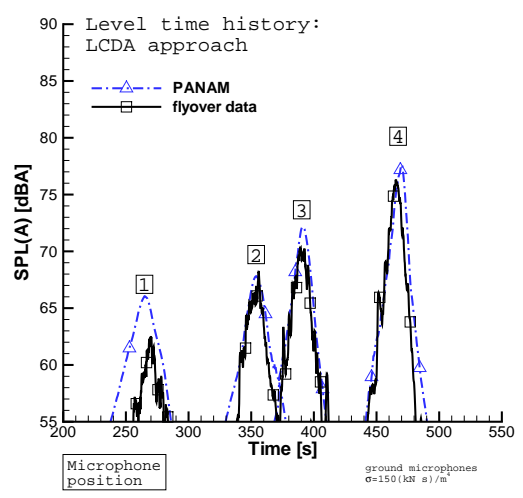

(a) level-time-history along flight ground track

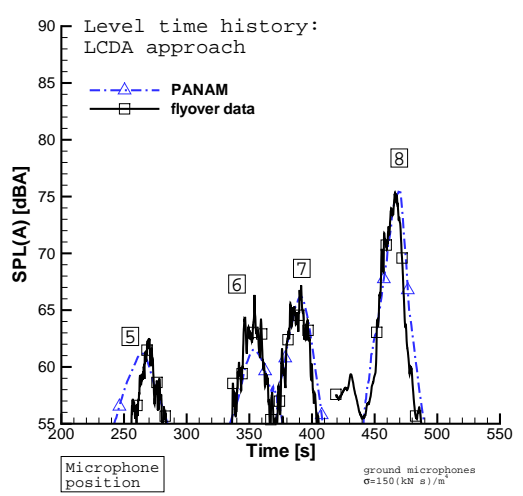

(b) level-time-history along sideline

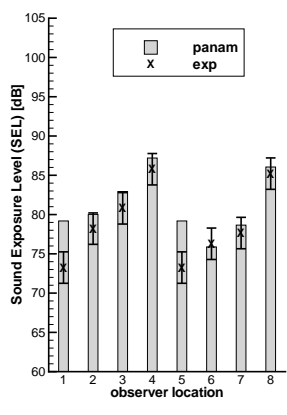

(c) A-weighted Sound Exposure Level (SEL)

Figure 4. Parchim Campaign: Continuous Descent Approach with Late Gear Extension (LCDA) 


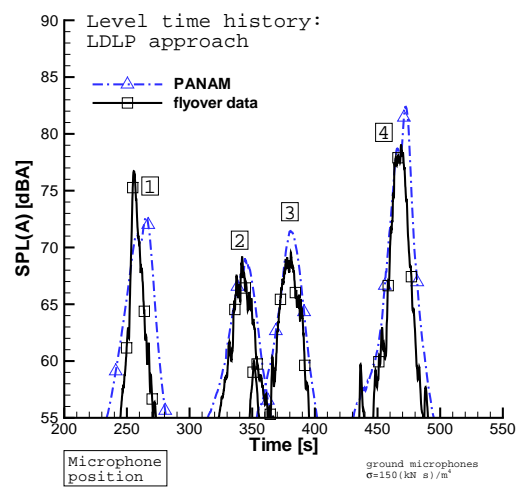

(a) level-time-history along flight ground track

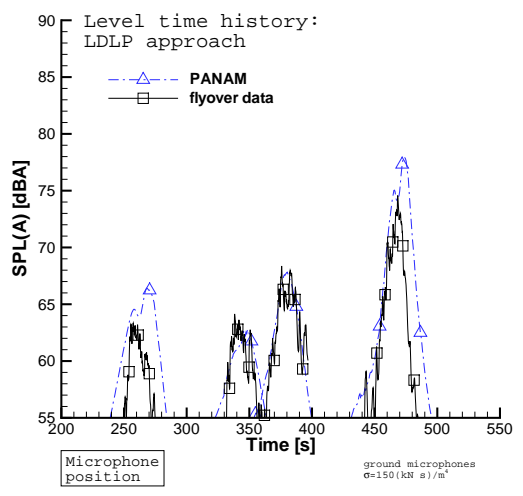

(b) level-time-history along sideline

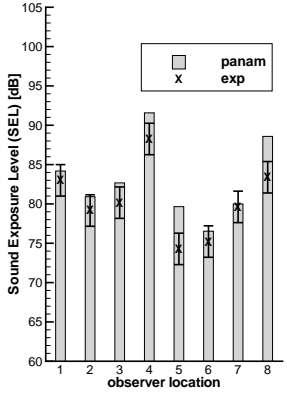

(c) A-weighted Sound Exposure Level (SEL)

Figure 5. Parchim Campaign: Low-Drag-Low-Power approach (LDLP)

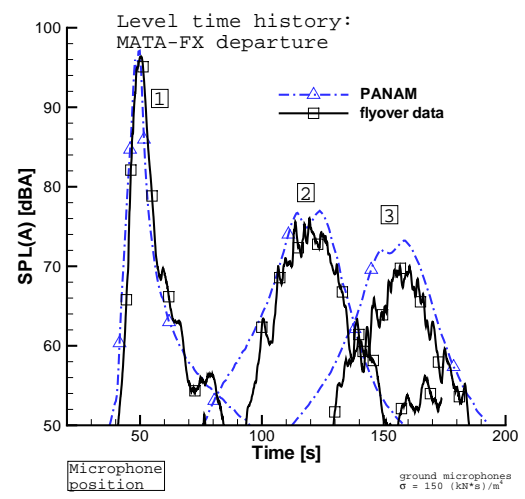

(a) level-time-history along flight ground track

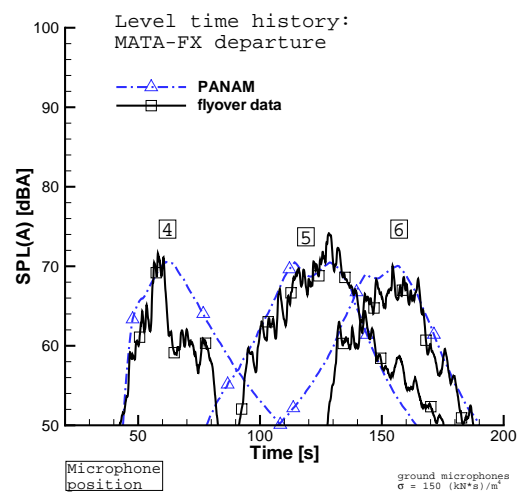

(b) level-time-history along sideline

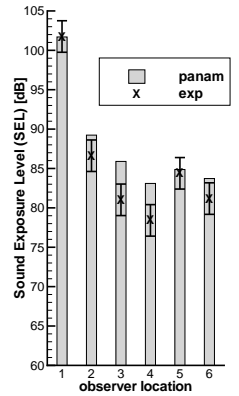

(c) A-weighted Sound Exposure Level (SEL)

Figure 6. Parchim Campaign: Departure level time history, Modified ATA Departure with Flexible Thrust (MATA-FX)

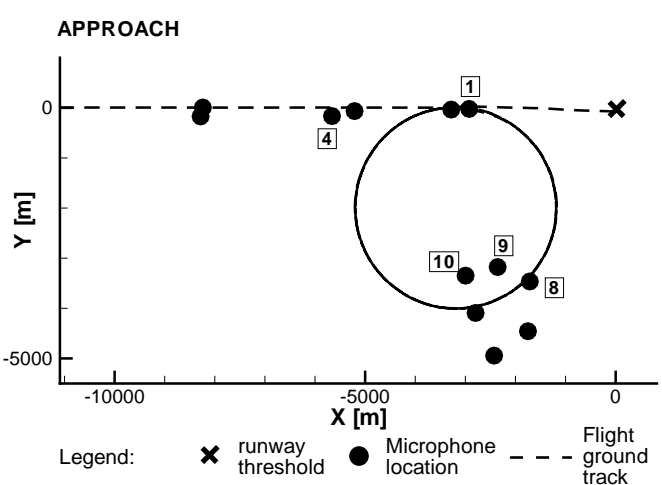

Figure 7. Braunschweig campaign 2009: Observer locations

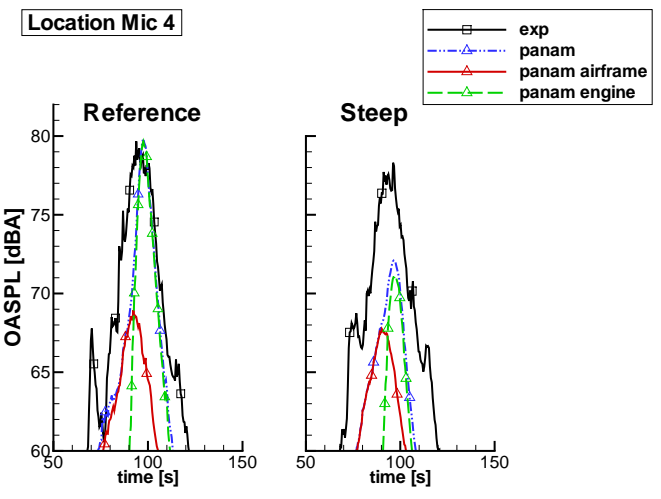

Figure 8. Braunschweig campaign 2009: Componential SPL(A) time-histories at observer location 4 


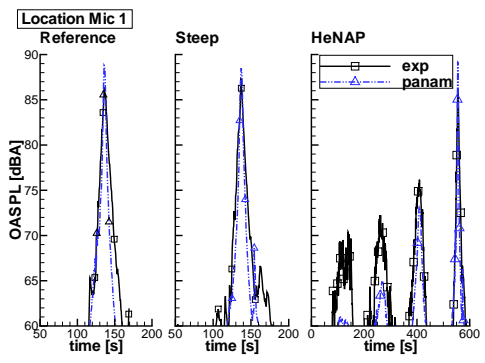

(a) mic 1

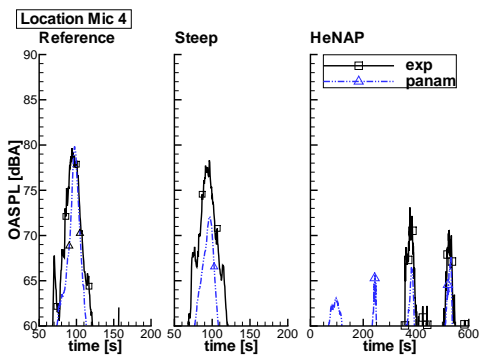

(b) $\operatorname{mic} 4$

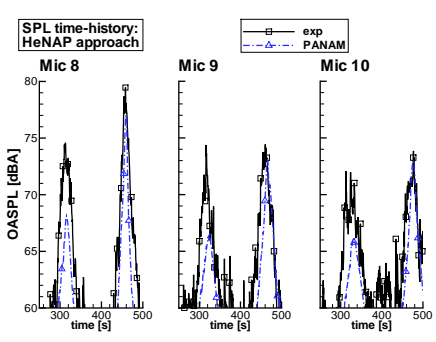

(c) mics 8-10 (HeNAP)

Figure 9. Braunschweig campaign 2009: SPL(A) time-histories
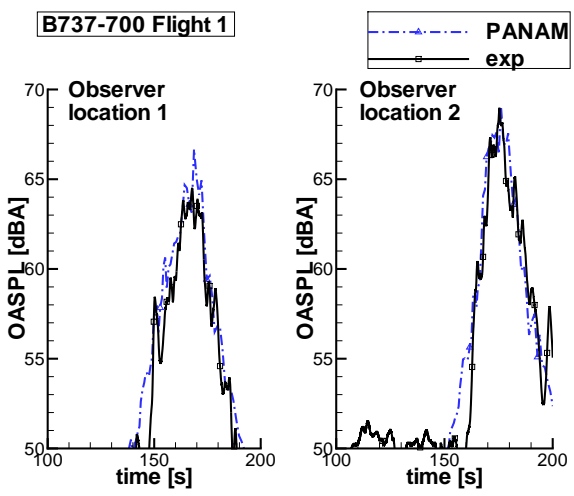

(a) level-time-history along flight 1
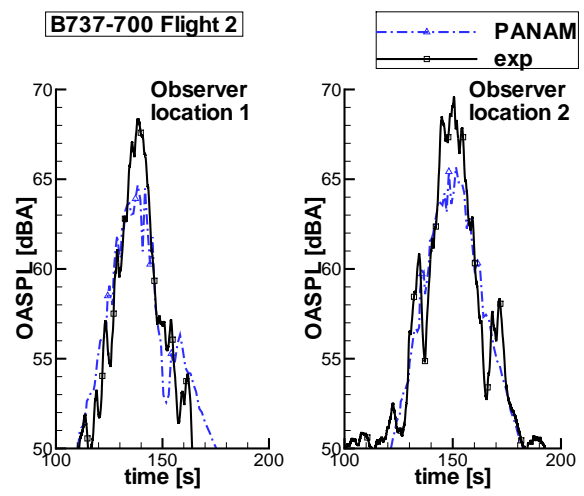

(b) level-time-history along flight 2

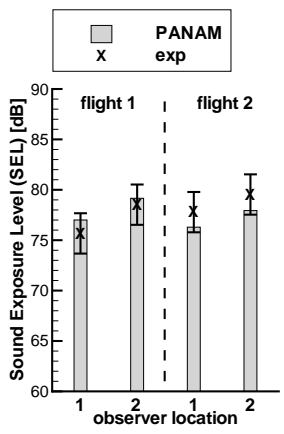

(c) A-weighted Sound Exposure Level (SEL)

Figure 10. Braunschweig Campaign 2010: Approach level time history, common approach procedures

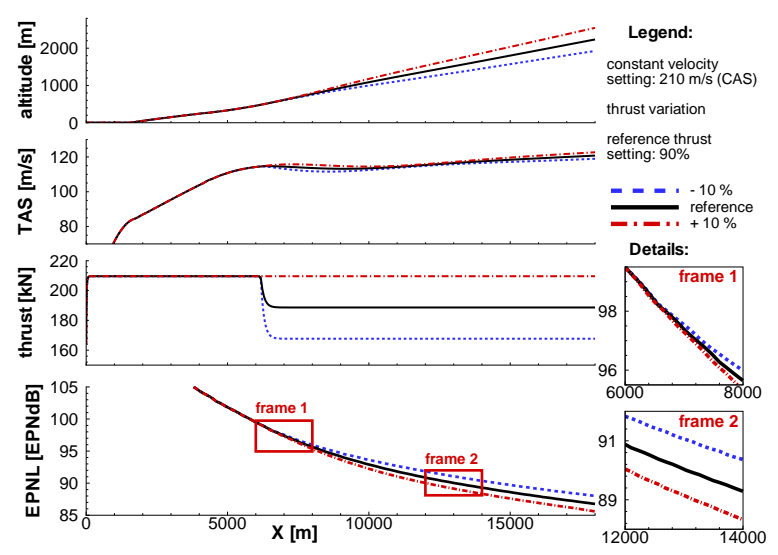

(a) thrust setting variation

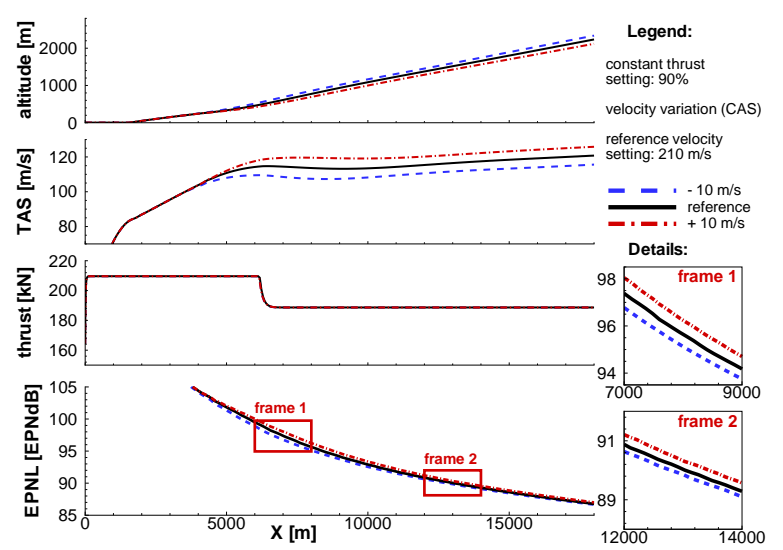

(b) velocity variation, CAS

Figure 11. Speed and thrust variation along departure: EPNL along flight ground track 
Componential max. SPL isocontours: leading edge devices vs. landing gear

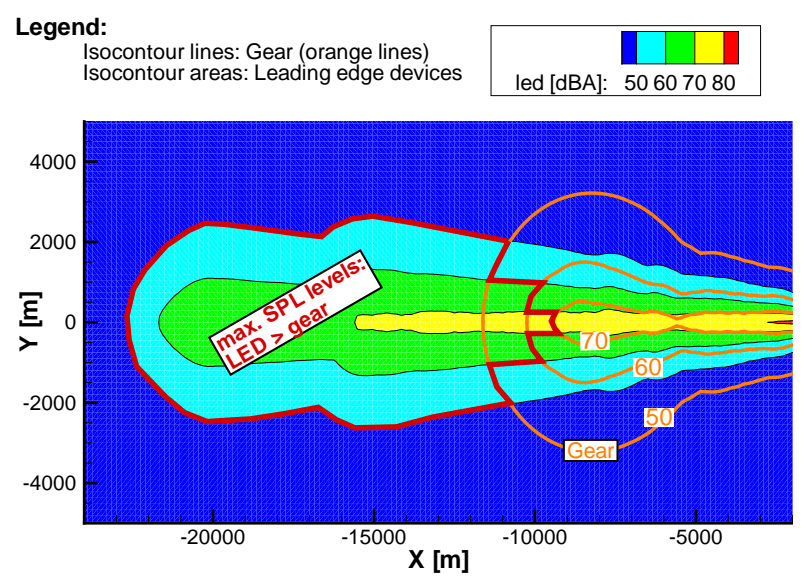

(a) Reference configuration: areas of dominating LED and landing gear ground noise

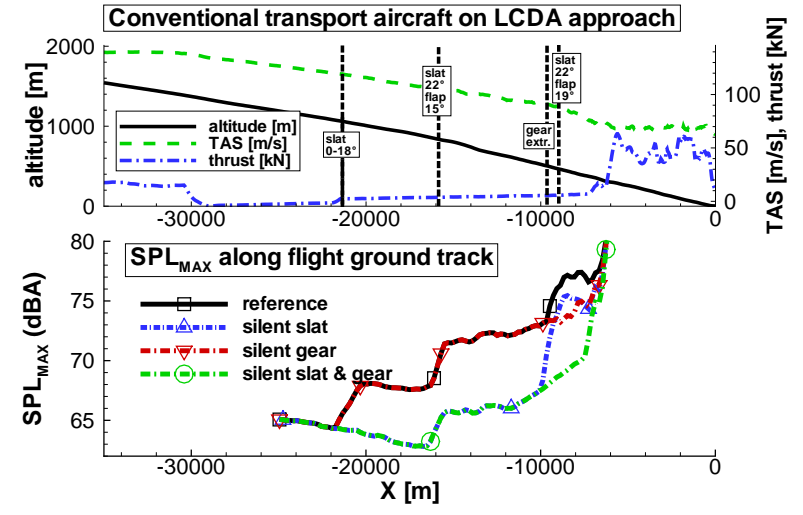

(b) Max. SPL along flight ground track: reference vs. lownoise aircraft (silent LED \& gear)

Figure 12. Conventional transport aircraft along LCDA approach: Impact of low-noise leading edge devices and low-noise landing gear

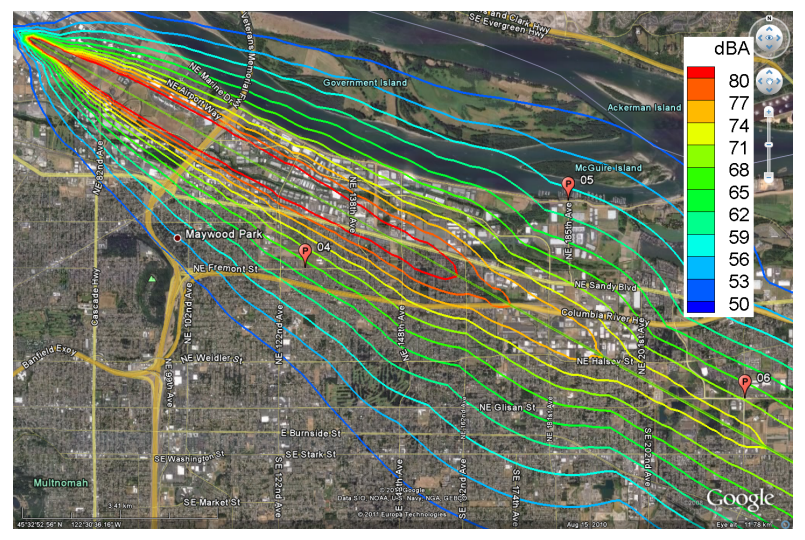

(a) reference a/c along LDLP approach

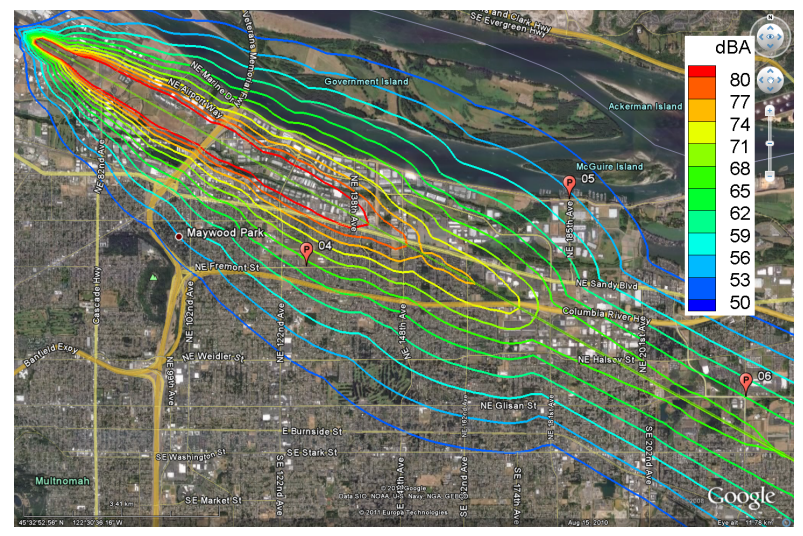

(b) low-noise LED a/c along LCDA procedure

Figure 13. Geographical noise impact visualisation ${ }^{52}$ : Max. SPL isocontour areas along simulated flights approaching KPDX runway 28L (airport noise measurement sites: red symbols) 


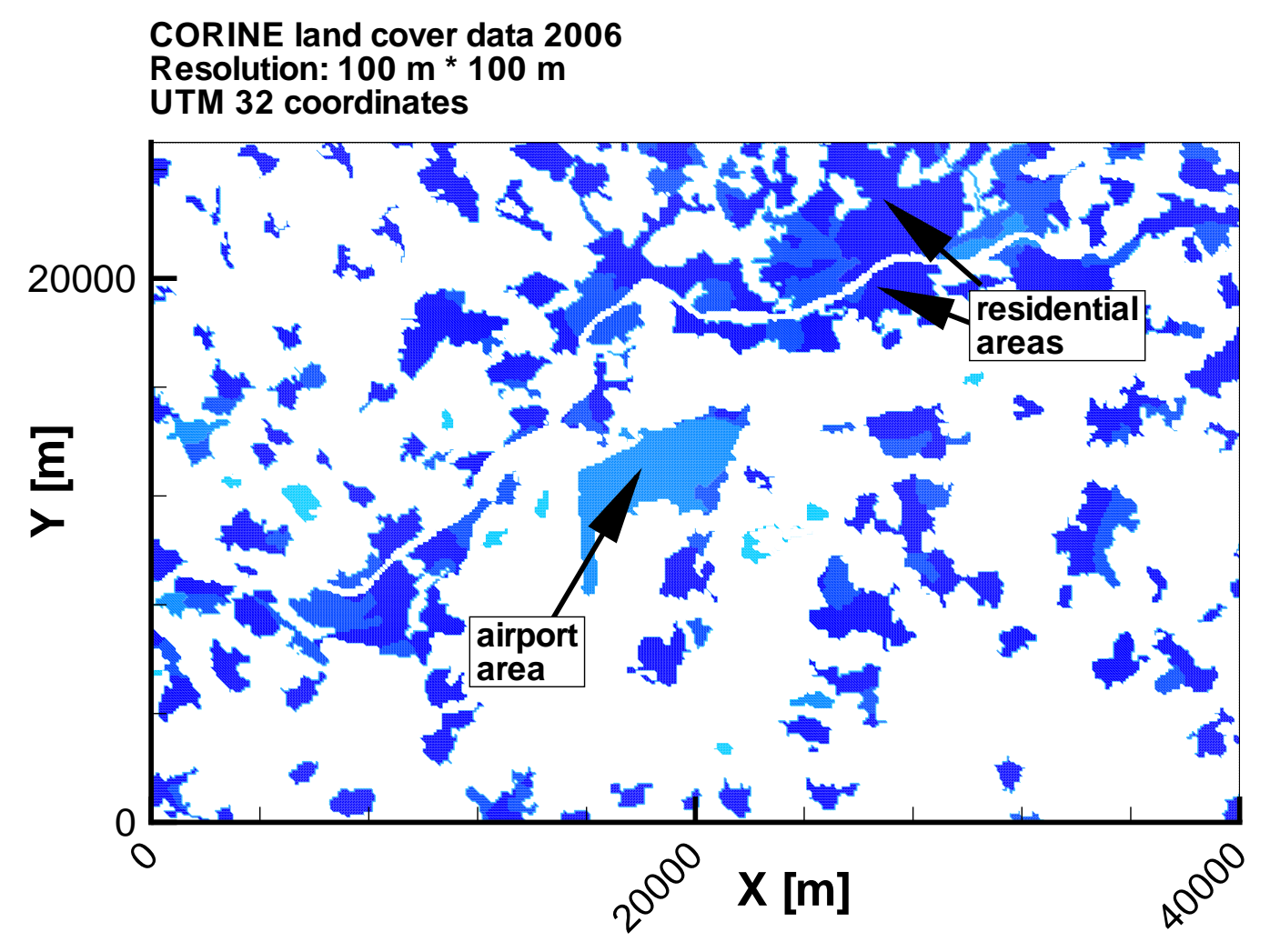

Figure 14. Frankfurt, Germany: Corine land cover data ${ }^{54}$ (blue: residential areas) 


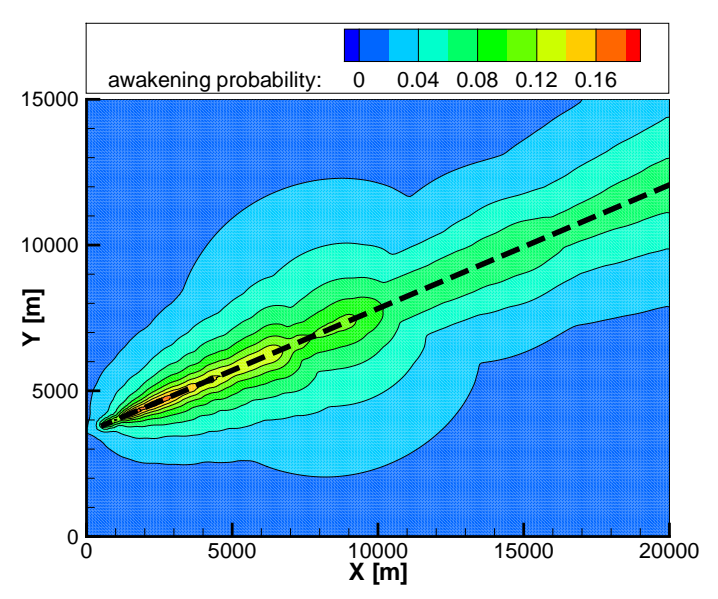

(a) awakening probability

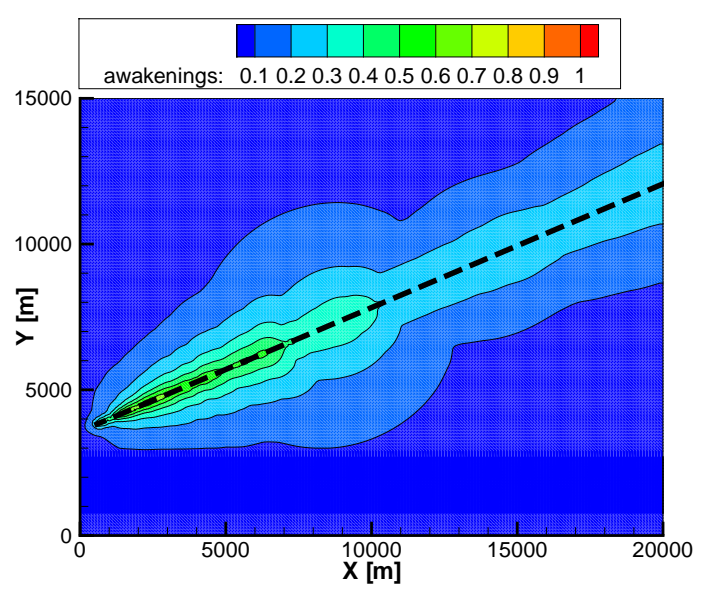

(b) awakenings for homogeneous population density (760 people $/ \mathrm{km}^{2}$

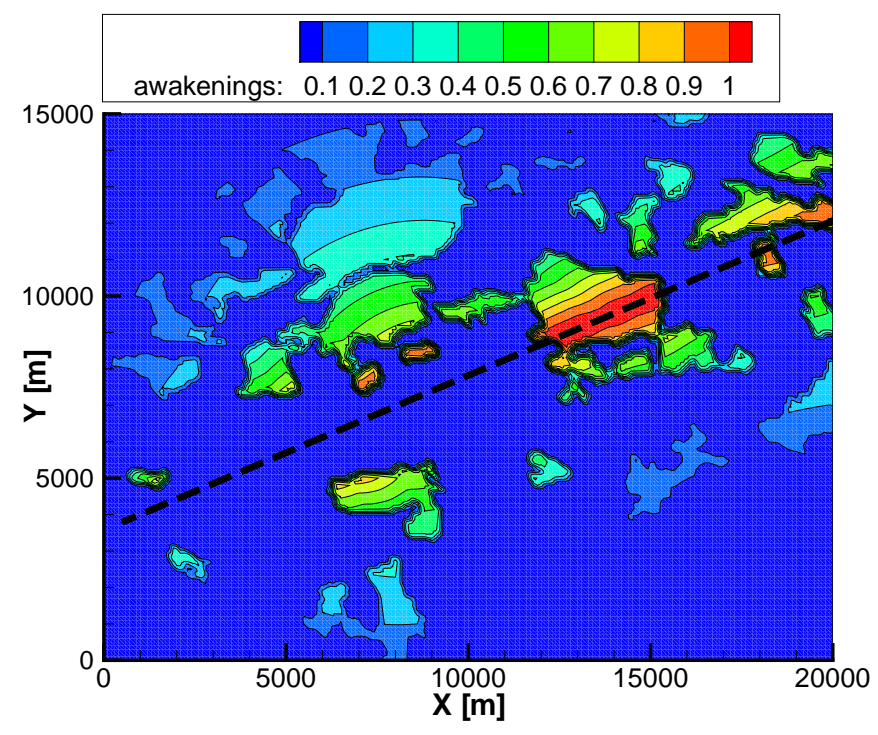

(c) awakenings for more realistic population distribution (2800 people $/ \mathrm{km}^{2}$, residential areas only)

Figure 15. Aircraft noise induced awakenings: aircraft with low-noise LED along LCDA 


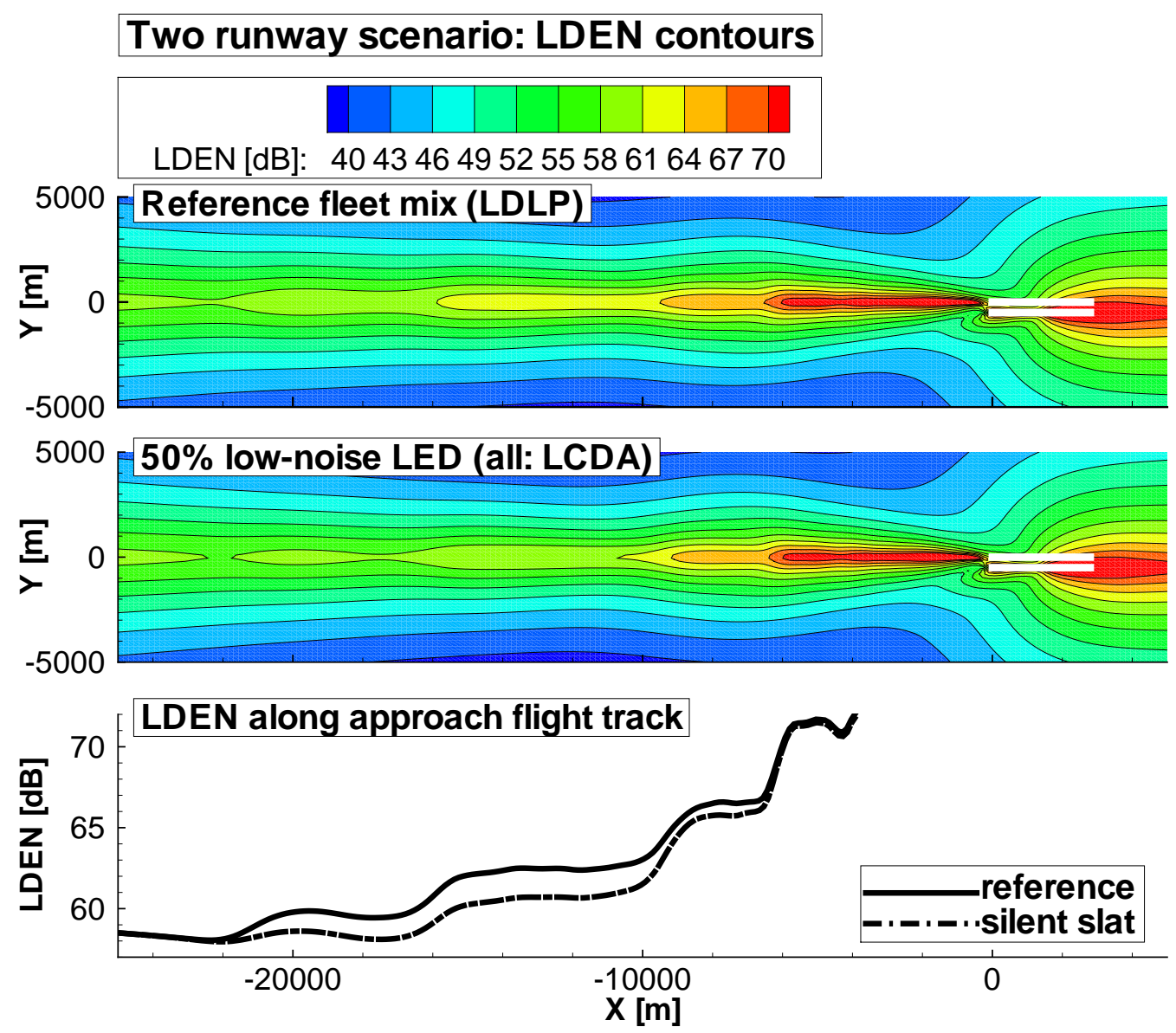

Figure 16. LDEN prediction: 2 runway scenario with $100 \%$ reference fleet mix (along LDLP) vs. $50 \%$ low-noise LED aircraft operation (all: along LCDA) 


\section{References}

${ }^{1}$ F.Farassat, J.H.Casper: Towards an Airframe Noise Prediction Methodology: Survey of Current Approaches, AIAA-2006-0210, 44th AIAA Aerospace Sciences Meeting and Exhibit, Reno, Nevada, January 9-12, 2006

${ }^{2}$ W.Dobrzynski: Almost 40 Years of Airframe Noise Research: What did we Achieve?, Journal of Aircraft, Vol. 47 (No. 2), pages 353-367, DOI: 10.2514/1.44457, March - April 2010

${ }^{3}$ Bundesministerium der Justiz: Erste Verordnung zur Durchfhrung des Gesetzes zum Schutz gegen Fluglrm (Verordnung ber die Datenerfassung und das Berechnungsverfahren fr die Festsetzung von Lrmschutzbereichen - 1.FlugLSV), Bundesgesetzblatt BGBl I, 27th December 2008

${ }^{4}$ European Civil Aviation Conference (ECAC): Methodology for Computing Noise Contours around Civil Airports, Vol. 1 and 2 (applications and technical guide), ECAC/CEAC Doc.29, 3rd Edition, December 2005

${ }^{5}$ Society of Automotive Engineers SAE, A-21 Aircraft Noise Measure Noise Aviation Emission Modeling: Procedure for the Computation of Airplane Noise in the Vicinity of Airports, SAE-AIR-1845, 1986

${ }^{6}$ U.Isermann, R.Schmid: Description and Calculation of Aircraft Noise (German), DLR-Forschungsbericht 2000-20, German Aerospace Center, ISSN 1434-8454, 2000

${ }^{7}$ S.Fidell, P.Schomer: Uncertainties in measuring aircraft noise and predicting community response to it, Noise Control Engineering Journal (NCEJ), Vol. 55 (No. 1), pages 82 - 88 , ISSN 0736-2501

${ }^{8}$ M.J.T. Smith: Aircraft Noise, Cambridge Aerospace Series, Cambridge University Press, ISBN 0-521-61699-9, 2004

${ }^{9}$ I.Jopson, D.Rhodes, P.Havelock: Aircraft noise model validation how accurate do we need to be?, Action on Environmental Noise, Institute of Acoustics Conference, Stratfordupon Avon, UK, November 2002

${ }^{10}$ J.R.Olmstead, G.G.Fleming, J.M.Gulding et al.:Integrated Noise Model (INM) Version 6.0 Technical Manual, U.S. Department of Transportation Federal Aviation Administration, Report No. FAA-AEE-02-01, January 2002, Washington, D.C., USA

${ }^{11}$ K.M.Eldred, R.L.Miller: Analysis of selected topics in the methodology of the Integrated Noise Model, Bolt Beranek and Newman Inc., Report No. 4413, Nov. 1980, USA

${ }^{12}$ S.Hartjes, H.G.Visser, S.J.Hebly: Optimization of RNAV Noise and Emission Abatement Departure Procedures, AIAA-2009-6953, 9th AIAA Aviation Technology, Integration, and Operations Conference (ATIO), September 2009, Hilton Head Island, South Carolina, USA

${ }^{13}$ S.Pietrzko, R.Buetikofer: FLULA - Swiss Aircraft noise prediction program, Innovation in Acoustics and Vibration, Annual Conference of the Australian Acoustical Society, 1315 November 2002, Adelaide, Australia

${ }^{14}$ U.Isermann: Calculation of Aircraft Noise in the Vicinity of Civil Airports by a Simulation Procedure (German), Dissertation, Report 7/1988, Max-Planck-Institut fuer Stroemungsforschung, 1988

${ }^{15}$ M.Pott-Pollenske, W.Dobrzynski, H.Buchholz, S.Guérin et al.: Airframe Noise Characteristics from Flyover Measurements and Predictions, AIAA-2006-2567, 12th AIAA/CEAS Aeroacoustics Conference (27th AIAA Aeroacoustics Conference), May 2006, Cambridge, Massachusetts, USA

${ }^{16}$ R.Koenig, J.Heider, M.Maierhofer: Aircraft Flight Procedure Design with Respect to Noise Abatement as well as Economical and Pilot Workload Aspects, Inter-noise Congress and Exposition on Noise Control Engineering 2005, August 2005, Rio de Janeiro, Brazil
${ }^{17}$ R.E.Gillian: Aircraft Noise Prediction Program Users Manual, NASA Langley Research Center, 1982

${ }^{18}$ M.R.Fink: Airframe Noise Prediction Method, FAA Research Report, FAA-RD-77-29, March 1977

${ }^{19}$ J.R.Stone, D.E.Groesbeck, C.L.Zola: Conventional profile coaxial jet noise prediction, AIAA Journal, 21(1) (1983), pp. 336-342

${ }^{20}$ M.F.Heidmann: Interim prediction method for fan and compressor source noise, NASA Technical Report TM-X71763, 1979

${ }^{21}$ J.Bridges, A.Khavaran, C.A.Hunter: Assessment of Current Jet Noise Prediction Capabilities, AIAA-2008-2933, 14th AIAA/CEAS Aeroacoustics Conference (29th AIAA Aeroacoustics Conference), 5 - 7 May 2008, Vancouver, British Columbia Canada

${ }^{22}$ E.Envia, D.L.Tweedt, R.P.Woodward et al.:An Assessment of Current Fan Noise Prediction Capability, AIAA2008-2991, 14th AIAA/CEAS Aeroacoustics Conference (29th AIAA Aeroacoustics Conference), 5 - 7 May 2008, Vancouver, British Columbia Canada

${ }^{23}$ N. Antoine et al.: A Framework for Aircraft Conceptual Design and Environmental Performance Studies, AIAA-20044314, 10th AIAA/ISSMO Multidisciplinary Analysis and Optimization Conference, August 2004, Albany, New York, USA

${ }^{24}$ E.D.Olson, D.N.Mavris: Aircraft Conceptual Design and Risk Analysis Using Physics-Based Noise Prediction, AIAA-2006-2619, 12th AIAA/CEAS Aeroacoustics Conference (27th AIAA Aeroacoustics Conference), May 2006, Cambridge, Massachusetts, USA

${ }^{25}$ N.Van Oosten: SOPRANO Presentation (PDF), SOPRANO Workshop, 21 - 22 June 2007, Madrid, Spain

${ }^{26}$ A.M.Rolt, K.G.Kyprianidis: Assessment of new aeroengine core concepts and technologies in the EU framework 6 NEWAC programme, paper icas-408, 27th International Congress of the Aeronautical Sciences (ICAS 2010), $19-24$ September 2010, Nice, France

${ }^{27}$ Y.Rozenberga, J.Bultéb: Fast Aircraft Noise Prediction Including Installation Effects for the Evaluation of Air Transport Systems, paper in08-0342, 37th International Congress and Exposition on Noise Control Engineering (inter-noise 2008), 26 - 29 October 2008, Shanghai, China

${ }^{28}$ M.Brunet, T.Chaboud, N.Huynh et al.: Environmental Impact Evaluation of Air Transport Systems Through Physical Modeling and Simulation, AIAA-2009-6936, 9th AIAA Aviation Technology, Integration, and Operations Conference (ATIO), September 2009, Hilton Head Island, South Carolina, USA

${ }^{29}$ W.Dobrzynski, M.Pott-Pollenske: Slat Noise Source Studies for Farfield Noise Prediction, AIAA-2001-2158, 7th AIAA/CEAS Aeroacoustics Conference, 28 - 30 May 2001, Maastricht, Netherlands

${ }^{30}$ L.Bertsch, W.Dobrzynski, S.Guérin: Tool Development for Low-Noise Aircraft Design, Journal of Aircraft, 47 (2), pages 694-699, DOI: 10.2514/1.43188, March - April 2010

${ }^{31}$ L.Bertsch, G.Looye, E.Anton, S.Schwanke: Flyover noise measurements of a spiraling noise abatement approach procedure, Journal of Aircraft, 48 (2), pages 436-448, DOI: 10.2514/1.C001005, March - April 2011

${ }^{32}$ K.-S.Rossignol: Development of an empirical prediction model for flap side-edge noise, AIAA-2010-3836, 16th AIAA/CEAS Aeroacoustics Conference, 7 - 9 June 2010, Stockholm, Sweden

${ }^{33}$ K.-S.Rossignol: Empirical Prediction of Airfoil Tip Noise, abstract of a paper proposed for presentation at the 17th AIAA/CEAS Aeroacoustics Conference, 6-8 June 2011, Portland, Oregon, USA 
${ }^{34}$ W.Dobrzynski et al.: Research into Landing Gear Airframe Noise Reduction, AIAA-2002-2409, 8th AIAA/CEAS Aeroacoustics Conference, June 2002, Breckenridge, USA

${ }^{35}$ W.Dobrzynski et al.: Experimental assessment of low noise landing gear component design, AIAA-2009-3276, 15th AIAA/CEAS Aeroacoustics Conference, 11 -13 May 2009, Miami, USA

${ }^{36}$ S.Guérin, U.Michel: Prediction of aero-engine noise: comparison with A319 flyover measurements, Tech. Rep., DLR, 2007, IB 92517-04/B3

${ }^{37}$ A.Moreau, S.Guérin, and S.Busse: A method based on the ray structure of acoustic modes for predicting the liner performance in annular ducts with flow, NAG/DAGA International Conference on Acoustics, Rotterdam, 23 - 26 March 2009

${ }^{38}$ J.M.Tyler, T.G.Sofrin: Axial flow compressor noise studies, SAE Transactions 70, 309-332, 1962

${ }^{39}$ W.Heinze: Ein Beitrag zur quantitativen Analyse der technischen und wirtschaftlichen Auslegungsgrenzen verschiedener Flugzeugkonzepte fuer den Transport grosser Nutzlasten (German), ZLR-Forschungsbericht 94-01, ISBN 3928628-14-3, 1994

${ }^{40}$ C.Liersch, M.Hepperle: A Unified Approach for Multidisciplinary Preliminary Aircraft Design, CEAS 2009 European Air and Space Conference, 26 - 29 Oct 2009

${ }^{41}$ M.Kunde, A.Bachmann, M.Litz: A dynamic data integration approach to build scientific workflow systems, Institute of Electrical and Electronics Engineers, International Workshop on Workflow Management (IWWM 2009), pages 27-33, May 2009

${ }^{42}$ Phoenix Integration: PHX ModelCenter and Analysis Server, http://www.phoenix-int.com

${ }^{43}$ M.Lummer: Maggi-Rubinowicz Diffraction Correction for Ray-Tracing Calculations of Engine Noise Shielding, 14th AIAA/CEAS Aeroacoustics Conference 2008, paper AIAA2008-3050

${ }^{44}$ C.Kott: Entwurf von laermarmen Flugzeugkonfigurationen unter Beruecksichtigung von Abschattungseffekten (German), final diploma thesis, German Aerospace Center in cooperation with Fachhochschule Aachen, Faculty of Aerospace Engineering, Aachen, Germany

${ }^{45}$ F.Deidewig: Ermittlung der Schadstoffemissionen im Unter- und Ueberschallflug, German Aerospace Center, DLR Forschungsbericht 98-10, Collogne, Germany, 1998

${ }^{46}$ G.Looye: Flight testing of autopilot control laws: fly the helix!, German Aerospace Congress 2009, paper DLRK-20091375
${ }^{47}$ G.Looye: Aircraft Mission Simulation for Environmental Analysis with Flight Test Validation, Presentation, DGLR workshop "Umweltfreundliches Fliegen", Braunschweig, Germany, June 16-17, 2010, http : //www.dglr.de (accessed 23 January 2011)

${ }^{48}$ L.Bertsch, G.Looye, T.Otten, M.Lummer: Integration and application of a tool chain for environmental analysis of aircraft flight trajectories, AIAA-2009-6954, 9th AIAA Aviation Technology, Integration, and Operations Conference (ATIO), September 2009, Hilton Head Island, South Carolina, USA

${ }^{49}$ M.Basner, A.Samel, U.Isermann: Aircraft noise effects on sleep: Application of the results of a large polysomnographic field study, Journal of the Acoustical Society of America, 119(5), pages 2272-2784, May 2006

${ }^{50}$ G.Mueller, M.Moeser: Taschenbuch der Technischen Akustik (German), 3rd edition, Springer Press, Berlin, ISBN 978-3-540-41242-7, 2004

${ }^{51}$ Position paper on dose response relationships between transportation noise and annoyance, European Communities, 2002

${ }^{52}$ Google: Google Earth software, http: \\earth.google.com

${ }^{53}$ W.Neise et al.: Forschungsverbund Leiser Verkehr, Bereich Leises Verkehrsflugzeug, Projekt 1600 Lrmoptimierte An- und Abflugverfahren (LAnAb), Final Project Report, Nov. 2007

${ }^{54}$ CORINE Land Cover (CLC2006), Umweltbundesamt (German Federal Environmental Agency), DLR-DFD 2009

${ }^{55}$ O.Schneider, S.Kreth, L.Bertsch: Towards a Quiet Short Take-Off and Landing Transportation System: Concept Evaluation and ATM Integration, IPLC-2010-0037, International Powered Lift Conference, October 2010, Philadelphia, Pennsylvania, USA

${ }^{56}$ Federal Aviation Agency - Capacity Modeling \& Analysis Group: SIMMOD - how it works, online version, http://www.tc.faa.gov/acb300/how_simmod_works.pdf (accessed 23 January 2011)

${ }^{57}$ L.Bertsch, O.Schneider, H.Hemmer, M.Hepperle, O.Macke: Process Implementation for the System Evaluation of new Low-Noise STOL Transportation Concepts, Paper No. 2, CEAS/KATnet II Conference on Key Aerodynamic Technologies, May 2009, Bremen, Germany

${ }^{58}$ C.Hange, D.Eckenrod: Assessment of a $C-17$ Flight Test of an ESTOL Transport Landing Approach for Operational Viability, Pilot Perceptions and Workload, and Passenger Ride Acceptance, AIAA Aerospace Sciences Meeting and Exhibit 2007, paper AIAA-2007-1398

${ }^{59}$ D.B. Hanson: Noise of counter-rotation propellers, Journal of Aircraft, 22 (7), pages 609-617, 1985 